\title{
Treatment of Metastatic Gastrointestinal Stromal Tumors (GIST): A Focus on Older Patients
}

\author{
Monika Dudzisz-Śledź ${ }^{1}$ Elżbieta Bylina ${ }^{1}$ Paweł Teterycz ${ }^{1}$ Piotr Rutkowski ${ }^{1}$
}

Accepted: 1 February 2021 / Published online: 2 March 2021

(c) The Author(s) 2021

\begin{abstract}
Gastrointestinal stromal tumors (GIST) originating in the Cajal cells are the most common mesenchymal neoplasms of the gastrointestinal tract. The median age of patients with this diagnosis is 65 years, and over $20 \%$ of cases affect people over the age of 70 years. The effectiveness and tolerability of systemic treatment with tyrosine kinase inhibitors in older patients with GIST seem to be similar to that in younger patients, but some studies have shown that treatment of older patients is suboptimal. Disability, frailty, comorbidities, and concomitant medications may influence treatment decisions, and toxicities also more often lead to treatment discontinuation. The known safety profile and oral administration route of the tyrosine kinase inhibitors used in GIST may allow maximization of treatment and the best efficacy, especially in older patients. This review summarizes the efficacy data for the systemic treatment of GIST, including data for older patients and from realworld experiences, if available and significant. The reported safety data and general rules for toxicity management, including appropriate patient selection and the need for careful monitoring during treatment, are also discussed.
\end{abstract}

\section{Key Points}

About $20 \%$ of gastrointestinal stromal tumors (GIST) affect patients aged $>70$ years.

Data on the efficacy and tolerability of tyrosine kinase inhibitors (TKIs), and the management of toxicities, in older patients with GIST are limited.

Older patients require careful selection for treatment as well as monitoring during treatment with TKIs so that toxicities may be detected early and appropriately managed.

\section{Introduction}

Gastrointestinal stromal tumors (GIST), originating from Cajal cells, are the predominant mesenchymal neoplasms of the gastrointestinal tract. The median age of patients with this diagnosis is 65 years. More than $20 \%$ of cases affect people over the age of 70 years [1]. Recognizing the molecular pathogenesis of GIST has led to the introduction of targeted therapies, primarily the small-molecule tyrosine kinase inhibitor (TKI) imatinib into clinical practice, and this has been a revolutionary breakthrough in the treatment of patients with GIST, initially in metastatic disease [2, 3]. The excellent prognosis in most localized cases can be contrasted with poor outcomes when imatinib therapy fails or is not feasible because of serious adverse events (SAEs) $[4,5]$. The latter can be especially true in older patients.

Therefore, understanding the molecular processes that govern this disease, and efficient clinical management of the side effects of GIST therapeutics, is crucial in the modern treatment of this group of neoplasms. While the effectiveness and tolerability of systemic therapies in older patients with GIST seem to be similar to that in younger patients, some studies have shown that the treatment of older patients is suboptimal. In general, the management of older patients with cancer is challenging, primarily because of heterogeneous health-related problems, including comorbidities,
Monika Dudzisz-Śledź

monika.dudzisz-sledz@pib-nio.pl

1 Department of Soft Tissue/Bone Sarcoma and Melanoma, Maria Sklodowska-Curie National Research Institute of Oncology, Roentgena 5, 02-781 Warsaw, Poland 
concomitant medications, cognition, disabilities, social issues, mood disturbances, and nutritional status. Such factors may influence treatment decisions, including treatment discontinuation when toxicities occur.

Systemic GIST treatment relies primarily on TKIs, which have a manageable safety profile. However, older patients were underrepresented in clinical trials of these drugs. Moreover, the data published from pivotal clinical trials usually present age as medians with ranges but do not summarize and interpret data separately for older patients. No universally accepted definition of the older population exists, and biological age is much more important than chronological age. Chronological age alone appears inadequate for characterizing the population enrolled in a clinical trial. The more appropriate predictor of clinical outcomes is frailty [6]. Additionally, the age of 65 years has been identified, along with multimorbidity and polypharmacy, as a significant risk factor for adverse drug reactions. However, chronological age is a much easier way to define this patient group, and the age of 70 years is the most commonly used cut-off point. It is recommended that clinical trials use the age of 65 years as the cut-off point for the older population [7]. Studies that evaluate the treatment of GIST in older patients have varying cut-off points for patient age, ranging from 65 to 75 years.

Treatment optimization seems to be the most important goal in the treatment of older patients. The known safety profile and oral administration route of TKIs presents an opportunity to maximize treatment and achieve the best efficacy, especially in this group of patients. It refers mostly to older patients able to receive full treatment. It is important to keep the patient's level of frailty in mind, as therapeutic decisions are more complicated in patients with frailty. It is also essential to find a balance between quality and quantity of life. This has become an important topic, especially in recent years. The European Medicines Agency developed a geriatric medicines strategy to ensure that medicines are appropriately studied in older patient populations and that studies are of high quality to improve the availability of information about the use of medicines in older people. Moreover, some clinical trials have assessed the influence of age and frailty on oncological treatment, including targeted therapies. This topic should be especially explored in the near future to increase our understanding so the knowledge can be transferred to everyday practice.

\section{Epidemiology}

GISTs are the most prevalent mesenchymal neoplasms of the gastrointestinal tract, with an incidence of 6.9/1,000,000/ year in the USA and 15/1,000,000/year across the EU $[8,9]$. They originate in the Cajal cells in the gastrointestinal tract, with a strong predominance in the stomach $(\sim 60 \%)$ and a lower predominance in the small and large intestine [4]. The median age of diagnosis is in the mid-60s, and as much as $21 \%$ of cases can occur after the age of 70 years.

\section{Biology}

An essential part of the development of GIST is the constitutional stimulation of several proliferation and survival pathways. This is usually accomplished by a mutation in one of two genes: KIT and platelet-derived growth factor receptor A (PDGFRA) [10]. The small subset of GISTs that does not harbor a mutation in those genes was historically called "wild-type" GISTs. Advancements in molecular biology allowed us to recognize that those "wild-type" tumors are usually characterized by succinyl dehydrogenase (SDH) deficiency due to epigenetic silencing of the $S D H C$ gene, mutations in NF1, or BRAF V600E mutations [11, 12]. "Wild-type" GISTs are predominant in pediatric patients and therefore outside the scope of this review. Molecular testing in the extensively evolving area of new drugs in GIST treatment should be recommended.

\subsection{KIT Mutations}

KIT (cluster of differentiation [CD]-117) is a transmembrane receptor with tyrosine kinase activity. It cooperates with its ligand - stem cell factor-and plays a physiological role in the survival, proliferation, and differentiation of hematopoietic cells, melanocytes, and gametes [13-15].

The distribution of activating KIT mutations in GISTs is not stochastic, as those deleterious variants occur mainly in exon 11 (about 60-75\%) and exon $9(5-15 \%)$ [16, 17]. These mutations lead to ligand-independent constitutional receptor activation and neoplastic transformation [18]. It is worth noting that clinically relevant differences exist between patients with exon 11 and exon 9 KIT mutations. Exon 9-mutated GISTs are less likely to respond to the standard dose of imatinib $400 \mathrm{mg}$ daily and most often require $800 \mathrm{mg}$ daily. This is true for both metastatic disease and adjuvant treatment [19-21].

Mutations in other KIT loci are rare and mainly associated with secondary resistance to imatinib (see Sect. 3.4).

\subsection{PDGFRA Mutations}

As mentioned, about $10-15 \%$ of GISTs harbor PDGFRA mutations. PDGFRA is another transmembrane tyrosine kinase phylogenetically related to, and with a structure similar to, KIT [22]. The most common regions of activating mutations in GIST include exon 18 (about 2-12\%) and exon 12 (up to $2 \%$ ) [16]. Some PDGFRA mutations are clinically 
significant as they predict primary resistance to imatinib (see Sect. 3.4).

\subsection{Downstream Pathways}

Downstream pathways for KIT and PDGFRA are similar and have a multimodal effect on a cell. The activation of these receptors leads to the propagation of the signal through the Src kinase, phosphatidylinositol 3-kinase, and mitogenactivated protein kinase (MAPK) [23-25]. The common downstream signaling causes mutual exclusion of KIT and PDGFRA mutations as the primary cause of GIST tumorigenesis [17].

\subsection{Mechanisms of Resistance}

Resistance to imatinib is a well-known phenomenon. Primary and secondary resistance can be distinguished. Mutations that hinder the ability of imatinib to connect to the KIT and PDGFRA protein-binding sites are responsible for primary resistance. The most common mechanism is the substitution of aspartic acid in codon 842 of PDGFRA into valine (D842V) [26]. Avapritinib, a newly developed TKI, is exceptionally active in patients diagnosed with this variant and has been approved by the US FDA for those patients [27, 28]. Mutations in exon 9 of KIT can also be considered a primary resistance mode as they show a worse response than most common exon 11 mutations [19-21]. Furthermore, "wild-type" GISTs are most often insensitive to standard therapies [29].

Secondary resistance can also be caused by amassing secondary point mutations in different regions of the KIT (such as exon 13,14,17, or 18) and PDGFRA genes [30]. Another interesting mechanism for secondary resistance is connected with fibroblast growth factor (FGF) and FGFreceptor (FGFR) [31]. It has been shown that crosstalk between KIT and FGFR can promote imatinib resistance by reactivating the MAPK signaling pathway.

\section{Clinical Trials}

Table 1 summarizes the main clinical trials of TKIs in the treatment of patients with advanced GIST.

\subsection{Imatinib}

The introduction of imatinib mesylate into clinical practice was a milestone in the treatment of GIST. Imatinib is a multitargeted TKI with activity against KIT and PDGFR. Prospective clinical trials in unresectable or metastatic GISTs have shown that complete responses (CRs) are only rarely observed (5-7\%). Partial remissions (PRs, 40\%) and stable disease (SD, 36\%) are the most common responses to treatment $[3,32,33]$. Longer use of imatinib in advanced GIST increases the percentage of partial remissions in patients with stabilization in the first months of treatment; at the same time, it is associated with a higher rate of progression. Overall survival (OS) in patients with advanced GIST is about 5 years, which is about four times longer than the historical data (median survival 12-15 months). The median progression-free survival (PFS) in imatinib-treated patients is $2-3$ years [42].

Treatment starts with an oral dose of imatinib $400 \mathrm{mg}$ once daily. It is currently recommended that the dose be increased to $800 \mathrm{mg}(2 \times 400 \mathrm{mg} /$ day $)$ at disease progression. The basic assessed parameters are the size of neoplastic lesions according to RECIST (Response Evaluation Criteria in Solid Tumors) criteria, assessment of the sum of the longest dimensions of measurable lesions, and the density of lesions (Choi criteria). Response should be carefully assessed. This is particularly important in differentiating between stabilization (inhibition of progression) and actual progression, as patients with disease stabilization evaluated according to the classic RECIST criteria benefit significantly from treatment (an effect similar to that observed in patients with partial remission).

During treatment with imatinib, some patients develop disease progression associated with drug resistance. A small proportion (10-15\%) of appropriately qualified patients (GIST CD117+) develop primary and early resistance during the first 6 months of treatment. Patients responding to treatment may develop secondary (acquired) resistance to imatinib with extended therapy. Disease progression occurs in approximately $40-50 \%$ of patients during the first $2-3$ years of imatinib treatment. Computed tomography scans may show a limited form of progression (e.g., a progression of one to two lesions with persistent regression of remaining metastases or the appearance of a growing nodule within a necrotic metastasis - the so-called tumor lump symptom). Mostly, however, images of multifocal progression are observed. The best responses to imatinib occur when the most common mutation in exon 11 is present, and much worse results occur with a mutation in exon 9 or no mutation in the KIT gene (sometimes associated with the presence of a certain mutation in the PDGFRA gene, especially D $842 \mathrm{~V}$ ) [17].

\subsection{Sunitinib}

The use of second-line TKIs should be considered for disease progression despite increasing the imatinib dose to 800 $\mathrm{mg}$ or if the patient is intolerant to imatinib. Other inhibitors that act at different target points in the metabolic pathway than the KIT exon 11 mutation may help overcome resistance to imatinib. Currently, the only approved second-line 


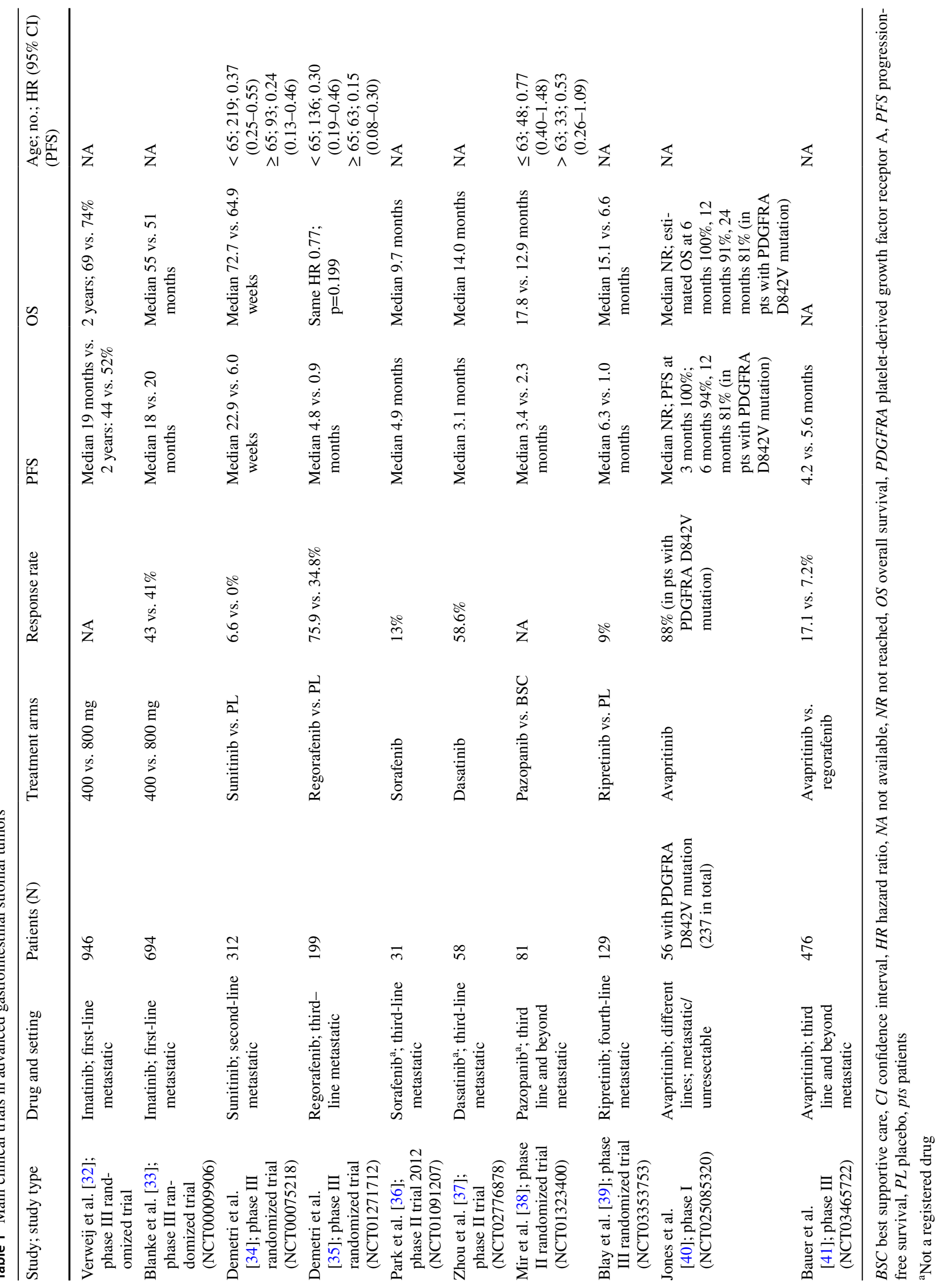


drug in the case of resistance to imatinib or drug intolerance is sunitinib malate. Sunitinib is a multitargeted TKI that acts on the KIT receptor tyrosine kinase, PDGFR, vascular endothelial growth factor receptor (VEGFR), and FLT3.

Available data indicate that about $40 \%$ of patients with imatinib-resistant GIST can achieve long-term responses, especially in the presence of the primary mutation in exon 9. The median time to progression in patients with GIST treated with sunitinib is 6-8 months. The results from a phase III, randomized, placebo-controlled, double-blind study showed that the median PFS during sunitinib treatment (starting dose of $50 \mathrm{mg}$ in the 4-week treatment, 2-week off schedule) was four times longer than that for placebo (22.9 vs. 6.0 weeks) $[34,43]$. Sunitinib should be started at a daily dose of $50 \mathrm{mg}$ in a 6-week schedule (4 weeks of active treatment and 2 weeks off). If toxicity is experienced, the daily dose of sunitinib can be reduced to 37.5 or $25 \mathrm{mg}$ and the treatment regimen break extended. An alternative continuous dosing regimen (37.5 $\mathrm{mg}$ daily without interruption) is widely accepted and seems to be more appropriate for TKIs [44, 45]. GIST genotype after imatinib resistance correlates with sunitinib activity. The median PFS and OS were significantly higher for patients with a primary $K I T$ exon 9 or wild-type KIT/PDGFRA mutation [45].

\subsection{Regorafenib}

Regorafenib, another multikinase inhibitor, has been approved for the treatment of hepatocellular carcinoma, metastatic colorectal cancer, and GIST. The recommended dose is $160 \mathrm{mg}$ taken orally once daily for the first 21 days of each 28-day cycle. Treatment is continued until disease progression or unacceptable toxicity. Regorafenib was first evaluated in an academic-initiated phase II clinical trial in patients with TKI-refractory GIST [46]. Regorafenib is the first drug in patients with GIST refractory to imatinib and sunitinib for which a phase III study demonstrated benefit in terms of PFS and disease control rate (DCR) (with a low percentage of objective responses). Regorafenib, in combination with optimal symptomatic treatment, significantly prolonged PFS in the progressive GIST population after all approved prior lines of treatment compared with the placebo group. The mean PFS in the regorafenib group was more than fivefold longer than in the placebo group [35]. Based on these results, regorafenib was approved (in the third line) for the treatment of metastatic or unresectable GIST after failure of or intolerance to imatinib and sunitinib.

\subsection{Ripretinib}

Ripretinib is a switch-control multikinase inhibitor with a dual mechanism of action that broadly inhibits KIT and PDGFRA kinases, including activity for wild-type KIT and
PDGFRA mutations, and multiple primary and secondary mutations associated with drug-resistant GIST. This molecule was assessed in a phase I first-in-human study in patients with GISTs and other advanced solid tumors. Preliminary efficacy was demonstrated in the phase II INVICTUS study (NCT03353753). Eligible patients were adults with GIST at Eastern Cooperative Oncology Group performance status 0-2 whose disease progressed on at least imatinib, sunitinib, and regorafenib or who had documented intolerance to any of these medications despite dose modifications. The patients were assigned to either ripretinib ( $n=$ $85)$ or placebo $(n=44)$. The median age of participants was 59 years (range 29-82) in the ripretinib group and 65 years (range 33-83) in the placebo group. In total, $32 \%$ of patients in the ripretinib group and $50 \%$ of those in the placebo group were aged $\geq 65$ years. The primary endpoint of this study was PFS. This study showed that ripretinib as a fourth or further treatment line significantly improved median PFS compared with placebo (6.3 vs. 1.0 months; hazard ratio [HR] 0.15 ; 95\% confidence interval [CI] 0.09-0.25; $p<$ 0.0001). Median OS was 15.1 months (95\% CI 12.3-15.1) in the ripretinib group and 6.6 months (95\% CI 4.1-11.6) in the placebo group (HR 0.36; 95\% CI 0.21-0.62) [39]. To date, there are no specific analyses from the studies with ripretinib dedicated to older patients.

Ripretinib is currently being assessed in comparison with sunitinib in the phase III study in patients with advanced GIST after treatment with imatinib (INTRIGUE; NCT03673501) [39, 47].

\subsection{Avapritinib}

Avapritinib, a type 1 kinase inhibitor, has been approved in the USA for the treatment of patients with unresectable or metastatic GIST harboring PDGFRA exon 18 mutations, including the PDGFRA D842V mutation, based on the results of the phase I NAVIGATOR study. In Europe, this drug has been approved for PDGFRA D842V GIST only [28, $48,49]$. NAVIGATOR was an open-label, phase I study that included dose-escalation and dose-expansion portions. The median age was 62 years. In the analysis published based on data from patients with PDGFRA D842V-mutation GISTs treated at any dose level, the confirmed overall response rate (ORR) was $88 \%$ (CR 9\%; PR 79\%; SD 13\%) [28]. The long-term efficacy data revealed an ORR of $95 \%$ (CR $13 \%$, PR $82 \%$ ) among 38 patients with PDGFRA D842V-mutant GIST treated with avapritinib $300 / 400 \mathrm{mg}$. The duration of response was 22 months, and the median PFS was 24 months. Median OS was not reached. The PFS and OS rates at 36 months were 34 and $71 \%$, respectively. The median age of those patients was 64 years (range 29-90) [28, 40]. To date, no specific analyses are available from the studies with avapritinib dedicated to older patients with GIST. 
Avapritinib was also assessed in the phase III, randomized, open-label VOYAGER study in patients with unresectable or metastatic GIST previously treated with imatinib and one or two other TKIs. The patients were randomized in a 1:1 ratio to treatment with avapritinib $(n=240)$ or regorafenib $(n=236)$. The study did not meet the primary endpoint of an improvement in PFS. PFS was 4.2 months in the avapritinib arm and 5.6 months in the regorafenib arm. This difference was not statistically significant. The ORR was $17 \%$ for the avapritinib group and $7 \%$ for the regorafenib group. The safety data for avapritinib were consistent with those reported in the previous study $[41,50]$.

\subsection{Other Agents}

Targeted agents, including dasatinib, nilotinib, cabozantinib, pazopanib, ponatinib, crenolanib, sorafenib, and others, have been investigated in patients with advanced GIST. Some of them are recommended after failure of approved therapies in certain situations. The data about the efficacy of the most important molecules are summarized in the following subsections. Numerous clinical trials assessing the efficacy and tolerability of various TKIs, immune checkpoint inhibitors, and other molecules are ongoing.

\subsubsection{Dasatinib}

Dasatinib has been approved by the FDA for the treatment of patients with chronic myeloid leukemia or acute lymphoblastic leukemia who have developed resistance or intolerance to imatinib. Dasatinib was investigated in TKI-naive GIST in a single-arm phase II clinical trial, but the trial was terminated early because of slow recruitment. Based on data from 43 eligible patients, the response rate at 4 weeks assessed using fluorodeoxyglucose-positron emission tomography was $67 \%$. The median PFS was 11 months [37]. The results of this study have become the basis for the off-label use of dasatinib in this indication, at the discretion of a physician [51]. As per National Comprehensive Cancer Network (NCCN) guidelines, dasatinib can be considered after failure of approved therapies for patients with a $P D G$ FRA D842V mutation [52].

\subsubsection{Pazopanib}

Pazopanib was assessed in the PAZOGIST study in patients with GIST. This was an open-label phase II trial and the first randomized study of pazopanib in patients with advanced or metastatic GIST for whom imatinib and sunitinib therapy had failed. The median age was 65 years (range 33-85) in the pazopanib group and 59 years (range 27-81) in the best supportive care group. Patients were randomly assigned to receive pazopanib plus best supportive care $(n=40)$ or best supportive care alone ( $n$ $=41$ ). If patients assigned to the best supportive care arm experienced disease progression, they could cross over to the pazopanib group [48]. The 4-month PFS rate based on central assessment was significantly higher in the pazopanib group $(44.3 \%)$ than in the control group (17.6\%). The results of the investigator-assessed 4-month PFS were consistent. Median investigator-assessed PFS was 3.4 months in the pazopanib arm and 2.3 months in the control arm. The median PFS in the patients who crossed over to the pazopanib arm was 3.5 months from pazopanib initiation [38].

\subsubsection{Sorafenib}

Sorafenib is a multikinase inhibitor with activity against KIT and PDGFRA and several other kinases. Based on the results of two single-arm phase II clinical trials, it is used to treat patients with advanced GIST after failure of or intolerance to imatinib and sunitinib in countries where regorafenib has not been registered or is not reimbursed. Sorafenib has not been approved for GIST treatment [53]. The results of both single-arm phase II clinical trials have demonstrated activity in patients with GIST after progression during therapy with imatinib and sunitinib, with a DCR at 24 weeks of $36 \%$. The median age of enrolled patients was 59 years (range 31-78) in the first study and 57 years (range 42-85) in the second. Median PFS in both trials was about 5 months, and OS was 9.7 and 11.6 months, respectively $[36,54]$.

\subsubsection{Cabozantinib}

Cabozantinib is a multitargeted TKI that targets KIT, VEGFR-2, MET, and AXL. The activity and safety of cabozantinib in patients with metastatic GIST after treatment with imatinib and sunitinib but no other KIT- or PDGFRdirected TKIs were assessed in the phase II, open-label, single-arm CaboGIST study (NCT02216578). The primary endpoint was PFS rate at 12 weeks, and the secondary endpoints included PFS, OS, ORR, DCR, duration of treatment, and safety assessed with Common Terminology Criteria for Adverse Events (CTCAE) version 4.0. The median age of patients enrolled in this study $(n=50)$ was 63 years (range 35-82). The median PFS at 12 weeks was $60 \%$ (95\% CI 45.0-74.0). DCR was $82 \%$ (95\% CI 69.0-91.0), with PR in $14 \%$ and SD in $68 \%$ of patients. The median PFS was 5.5 months (95\% CI 3.6-6.0), and the median OS was 18.2 months (95\% CI 14.3-22.3). The mutational analysis was conducted in archival tissue samples from 37 patients. The most frequently found mutations were KIT mutations $(83.3 \%)$ [55]. 


\subsubsection{Ponatinib}

This novel multitargeted TKI was tested against a variety of KIT-mutant GIST. Ponatinib has shown activity against the KIT exon 17 D816-mutant kinases [56]. This molecule was assessed in a phase II single-arm clinical study in patients with unresectable and metastatic GIST after failure of prior TKI therapy $(n=45)$ (NCT01874665). Patients were enrolled in two cohorts based on the presence (A) or absence (B) of primary mutations in KIT exon 11 . The median age of patients was 59 years. The clinical benefit rate $(\mathrm{CR}+\mathrm{PR}+\mathrm{SD})$ in patients with KIT exon 11 mutations at 16 weeks was 37\% [57]. This inhibitor was assessed in another phase II study, the POETIG trial (NCT03171389). Given the dose-dependent toxicity profile of ponatinib, the authors assessed the efficacy and tolerability of a reduced dose in patients with GIST pretreated with other TKIs. The results of this study, published by Falkenhorst et al. [58], revealed notable activity of lower-dose ponatinib in those patients $(n$ $=39$ ), with a safety profile comparable to that of other TKIs used in GIST. The clinical benefit rate was 35\% (95\% CI 15.4-59.2). The median PFS was 86 days [58].

\subsubsection{Nilotinib}

Nilotinib is a selective and potent TKI that targets BCRABL, c-KIT, PDGFR, and other kinases. Nilotinib was assessed in the first and further treatment lines in advanced GIST. Despite not being registered for that indication, it can be used in some situations after the failure of other registered TKIs [52].

In the randomized phase III clinical study, nilotinib was compared with best supportive care with or without imatinib or sunitinib in patients with GIST resistant or intolerant to imatinib and sunitinib $(n=249)$. The primary endpoint was PFS. The mean age of all patients was about 58 years. Median PFS was similar between both treatment arms (nilotinib 109 days, best supportive care 111 days; HR 0.90; $p=$ 0.56 ). The analysis based on the investigator's assessment in the intent-to-treat population revealed a significantly higher median PFS with nilotinib (119 vs. 70 days; $p=0.0007$ ). Post hoc subset analyses in patients with progression and only one prior regimen revealed a significant difference in median OS: 405 days for nilotinib and 280 days for the comparator $(p=0.02)$ [59].

Nilotinib was assessed in the first-line setting in metastatic GIST compared with imatinib in a randomized phase III open-label study (NCT00785785). This study did not meet the primary endpoint. The 2-year PFS was higher in the imatinib group than in the nilotinib group (59.2 vs. 51.6\%, respectively). Based on these study results, nilotinib cannot be recommended for use in the first-line setting in advanced GIST [60].
Montemurro et al. [61] assessed nilotinib in a retrospective analysis of 52 patients with advanced GIST resistant to imatinib and sunitinib. Median PFS and OS were 12 weeks and 34 weeks, respectively [61].

Another study, by Cauchi et al. [62], evaluated nilotinib in 13 patients with advanced GIST previously treated with imatinib and sunitinib. The median age of patients was 63 years. The study was closed early because of insufficient clinical benefit. Based on the molecular testing and treatment results, the authors concluded that nilotinib may provide benefit to specific subsets of advanced GIST with exon 17 mutations [62].

\subsubsection{Crenolanib}

Crenolanib is a TKI with activity against PDGFR and FLT3. Crenolanib has shown activity in GIST with a PDGFRA D842V mutation resistant to imatinib [63]. This molecule was assessed in a phase II study (NCT01243346) [64] and is currently being tested in a randomized, double-blinded, placebo-controlled phase III trial in patients with advanced or metastatic GIST with a D $842 \mathrm{~V}$ mutation in the PDGFRA gene (CrenoGIST; NCT02847429) [65].

\section{Real-World Experience with a Focus on the Older Patient Population}

The history of imatinib treatment in GIST is about 20 years long. During this period, a large amount of real-world data on its safety and efficacy has been accumulated. At the same time, the number of papers regarding treatment in the older patient population is limited. This is concerning given that, in the prognostic nomogram based on the Surveillance, Epidemiology, and End Results program database, age was an independent prognostic factor for both OS and cancerspecific survival (CSS) [66]. In this comprehensive analysis, patients aged 65-79 years had an HR for CSS equal to 1.568 (95\% CI 1.155-2.199) compared with patients aged $<50$ years. Simultaneously, patients aged $>80$ years had an HR for CSS equal to 1.639 (95\% CI 1.265-2.207). Similar correlations were shown in a retrospective analysis of multicenter German data, where age $>50$ years was linked to a worse prognosis in terms of OS, disease-free survival (DFS), and disease-specific survival (DSS) [67]. This may be related to an observation by Farag et al. [68] who, in a retrospective study based on the Dutch GIST registry, reported that older patients were less likely to undergo surgery for localized GIST and to receive adjuvant treatment, regardless of comorbidity and performance score.

No data/subgroup analyses on older patients are available from the registration trials in the patients with advanced GIST treated with TKIs. The quality-of-life data 
from clinical trials are very limited and show no impairment of these parameters in the general GIST population treated with imatinib rechallenge and no difference between patients treated with regorafenib and best supportive care in the GRID trial [69, 70].

To date, the largest real-world study concerning the systemic treatment of advanced GIST in older patients was based on the Polish GIST registry. This study included 139 patients aged $>70$ years who started imatinib for metastatic/ locally unresectable disease, comparing them with 517 younger patients [71]. In this report, Rutkowski et al. [71] found no differences in PFS with imatinib between analyzed groups with a median first-line PFS of 44.9 months (95\% CI 32.7-64.1) in older and 38.5 months (95\% CI 30.6-43.5) in younger patients $(p=0.4)$. DSS (medians not reported) also did not differ $(p=0.3)$, nor did baseline mutational status and laboratory test results. However, significant differences were seen in the frequency of comorbidities (more common in older patients) and the probability of permanent dose reductions. OS was also shorter in older patients (50 months; 95\% CI 42.6-71.2) than in those aged $<70$ years (81 months; 95\% CI 70.2-91.7; $p<0.001$ ). The median second-line PFS and OS (sunitinib) reached 9.7 and 21.5 months, respectively, and were not significantly different from those in younger patients $(p=0.7$ and 0.05 , respectively) [71].

The Dutch registry-based study reported by Farag et al. [68] also focused on metastatic disease. In this paper, 36 patients aged $>75$ years were diagnosed with metastatic GIST. Of these, 31 received first-line treatment. Similar to other studies, no statistically significant PFS differences were seen between older and younger patients, yet the OS differed greatly. Median PFS reached 24 (95\% CI 13.3-34.7) and 33 months (95\% CI 27.4-38.6; $p=0.1$ ), whereas median OS reached 34 (95\% CI 13.0-55.0) and 59 months (95\% CI not available; $p=0.01$ ) for older and younger patients, respectively. No statistically significant differences in qualification to the further treatment lines between the age groups were reported, possibly because of the small sample size [68].

Mandel et al. [72] reported a group of 85 patients aged $>65$ years with GIST. In this analysis, 26 patients had metastatic disease. The 5-year OS rate in the metastatic setting reached $80 \%$ [72]. In an interesting study, Italiano et al. [73] evaluated survival in a cohort of 44 older patients with advanced GIST and measured the imatinib concentration in 24 of them. Researchers reported a median PFS of 34.4 months (95\% CI 11.5-57.4) and a median OS of 50.3 months (95\% CI 37-63.5). Moreover, they identified ECOG performance status $<2$ as an independent positive predictor for OS and suggested that age only slightly influenced imatinib pharmacokinetics [73]. A small analysis by Tham et al. [74] included 18 patients aged $>65$ years; 13 had metastatic disease. The median PFS and OS in patients with metastatic disease was 33 and 37.6 months, respectively, and did not differ from results in younger patients. The analysis showed that, in older patients, the comorbidities were associated with a higher risk of disease recurrence $(p=0.046)$ and with the shorter OS $(p=0.005)$ [74].

Yang et al. [75] analyzed the clinicopathological and prognostic data from 1846 patients with primary gastric GIST. They compared data from younger patients $(n=1264)$ and those aged $\geq 65$ years $(n=582)$. The analysis included patients treated from 1998 to 2015, and many were treated before imatinib became the standard of adjuvant therapy. They found a higher incidence of comorbidities (40.7 vs. $23.5 \% ; p=0.011)$ and postoperative complications (14.4 vs. $9.7 \% ; p=0.031$ ) in older patients. Fewer patients from the older group received adjuvant therapy (30 vs. $66.8 \% ; p$ $=0.001)$. Older patients had a higher rate of tumor necrosis and worse DFS and OS than younger patients [75].

Moreover, many authors emphasize that comorbidities, including secondary malignancies, are more common in older patients $[71,76,77]$. In the report by Vassos et al. [77], $42 \%$ of the study cohort $(n=86)$ were diagnosed with secondary malignancy. The mean age for such a diagnosis was 70 years. Smith et al. [76] showed that older age was significantly $(p<0.0001)$ correlated with additional cancer, which translated into worse OS. The adjusted HR for developing secondary malignancy within 6 months of GIST diagnosis versus no additional malignancy reached 1.55 (95\% CI $1.05-2.30 ; p=0.042$ ) [76].

It may be worth noting that the cut-off point for "older age" differs between studies, ranging from 65 to 75 years. Therefore, any clinical decision making, especially in this subgroup of patients, must be individualized and must consider both the heterogeneity of the discussed population and the patient's preferences. Moreover, several studies on cancer highlight that using geriatric assessment (with specific tools such as the Comprehensive Geriatric Assessment [CGA] or age-adjusted Charlson Comorbidity Index) improves decision making and may predict the treatment toxicity $[78,79]$.

\section{Management of the Adverse Events of Tyrosine Kinase Inhibitors (TKIs) in Older Patients}

The treatment of older patients has become increasingly important over the last 10 years, especially because of the high vulnerability of this patient population. The manageable safety profile and the oral administration route of TKIs represent significant advantages for the treatment of older patients. Data about the incidence and management of adverse events (AEs) in older patients with GIST receiving 
imatinib and other kinase inhibitors are limited. The general rules for toxicity management should be applied, with an emphasis on an individualized approach for each patient. AEs can be managed through dose reductions or dose interruptions and supportive medications. Maximizing drug exposure with treatments for GIST is essential, as this has a proven impact on treatment outcomes and prolonged disease control. Dose adjustments should be carefully considered. Imatinib is the drug most frequently used for GIST treatment and is used in both perioperative and metastatic settings, and this treatment is associated with a known toxicity profile.

The predictive factors of TKI toxicities in older and fragile patients with GIST are unknown. Some data have been published for TKIs in general or for other indications, which can be considered useful for TKI use in older patients with GIST.

The multicenter PRETOXE study (NCT02751827) published by Lebreton et al. [80] in 2019 included three cohorts of patients aged $>70$ years with different advanced solid tumors treated with TKIs according to the approved indications $(n=371)$. The median age of enrolled patients was 74 years (range 70-88). The most frequently used TKIs were everolimus, erlotinib, pazopanib, gefitinib, and regorafenib. The dose administered was lower than approved for specific indications in $32.1 \%$ of patients. Severe toxicities were reported in almost 30\% of patients. Multivariate analysis indicated that independent predictive factors of severe toxicity included female sex, three or more concomitant medications, and the anti-angiogenic activity of TKIs [80].

The cardiovascular toxicity of angiogenesis inhibitors in the treatment of malignancies is a well-known complication. The increased risk of hypertension, arterial thromboembolism, cardiac ischemia, and cardiac dysfunction is associated with either TKIs or direct VEGF inhibitors. The analysis of clinical data by Hamnvik et al. revealed that factors predictive of hypertensive response to anti-VEGF inhibitors included previously existing hypertension, age $\geq$ 60 years, and body mass index $\geq 25 \mathrm{~kg} / \mathrm{m}^{2}$. The analysis included 1120 patients with renal cell carcinoma, hepatocellular cancer, GIST (12.5\%), and other sarcomas treated with pazopanib, sorafenib, and sunitinib. It is important to emphasize that this analysis confirmed that the development of hypertension predicted improved survival (HR $0.76 ; 95 \%$ CI 0.65-0.89) [81, 82].

Table 2 summarizes the most frequent AEs reported in registration trials.

\subsection{Imatinib}

It is important to note that the concentrations and consequently the tolerability and efficacy of imatinib may be affected by drug-drug interactions, the genetic variability of metabolizing or drug-resistance enzymes, and compliance [83]. Clinical trials with imatinib included over $20 \%$ of patients aged $\geq 65$ years. No significant agerelated pharmacokinetic differences have been observed in adult patients [83]. Population pharmacokinetics in patients with chronic myeloid leukemia indicated a small effect of age on the volume of distribution (12\% increase in patients aged $>65$ years) [84]. This change is not clinically significant, and there is no need for dose modification in older patients [85]. Of interest, no important effects of concomitant medications were observed. However, in routine practice, multidrug interactions related to polypragmasia, often occurring in older patient populations, can be problematic. Doses of different TKIs may be adjusted in a rational way with the use of therapeutic drug level monitoring [86].

In 2011, D'Ambrosio et al. [87] presented the results of imatinib treatment in 23 patients with GIST. The mean age of patients included in this analysis was 79 years (range $75-90)$. The starting dose of imatinib was $400 \mathrm{mg} /$ day in $20(87 \%)$ and $800 \mathrm{mg} /$ day in three $(13 \%)$ patients. If disease progressed on the 400-mg dose, dose escalation was attempted. Patients were followed until progression or death, and toxicities were reported based on CTCAE version 3.0. The median number of comorbidities was two. Imatinib was generally well-tolerated, and efficacy was comparable to that in younger patients. Three patients received doses of $800 \mathrm{mg}$, and doses of $600 \mathrm{mg}$ were well-tolerated in seven patients (30\%). In total, 11 (48\%) grade 3 and 4 toxicities were reported in patients treated with the 400-mg dose: edema (four cases), rash (three cases), and anemia (four cases). The following AEs were reported in patients treated with $800 \mathrm{mg}$ : edema (five cases), ocular toxicities (three cases), and anemia (five cases). No imatinib-related deaths were observed. No patients discontinued treatment because of AEs. The median PFS was 29 months, and the median OS was 49 months. Four patients aged $>80$ years received imatinib for more than 4 years. The authors concluded that older patients with GIST treated with imatinib should be carefully monitored, especially regarding toxicities requiring dose reductions such as fluid retention (edema), anemia, and ocular toxicities [87]. Farag et al. [68] observed similar AEs related to imatinib treatment and dosage in the older and younger groups.

High-dose imatinib is associated with severe drug-related side effects in many cases. Imatinib can cause serious hematological and nonhematological adverse reactions. In some cases, even a standard dose of $400 \mathrm{mg} /$ day is intolerable. The most common side effects of imatinib are fluid retention and edemas (particularly periorbital), diarrhea, nausea and vomiting, muscle cramps in fingers and feet, fatigue, abdominal pain, and rash. SAEs such as abnormal liver function test, gastrointestinal bleeding, lung toxicity, and hematologic adverse reactions have been rarely reported [88]. 


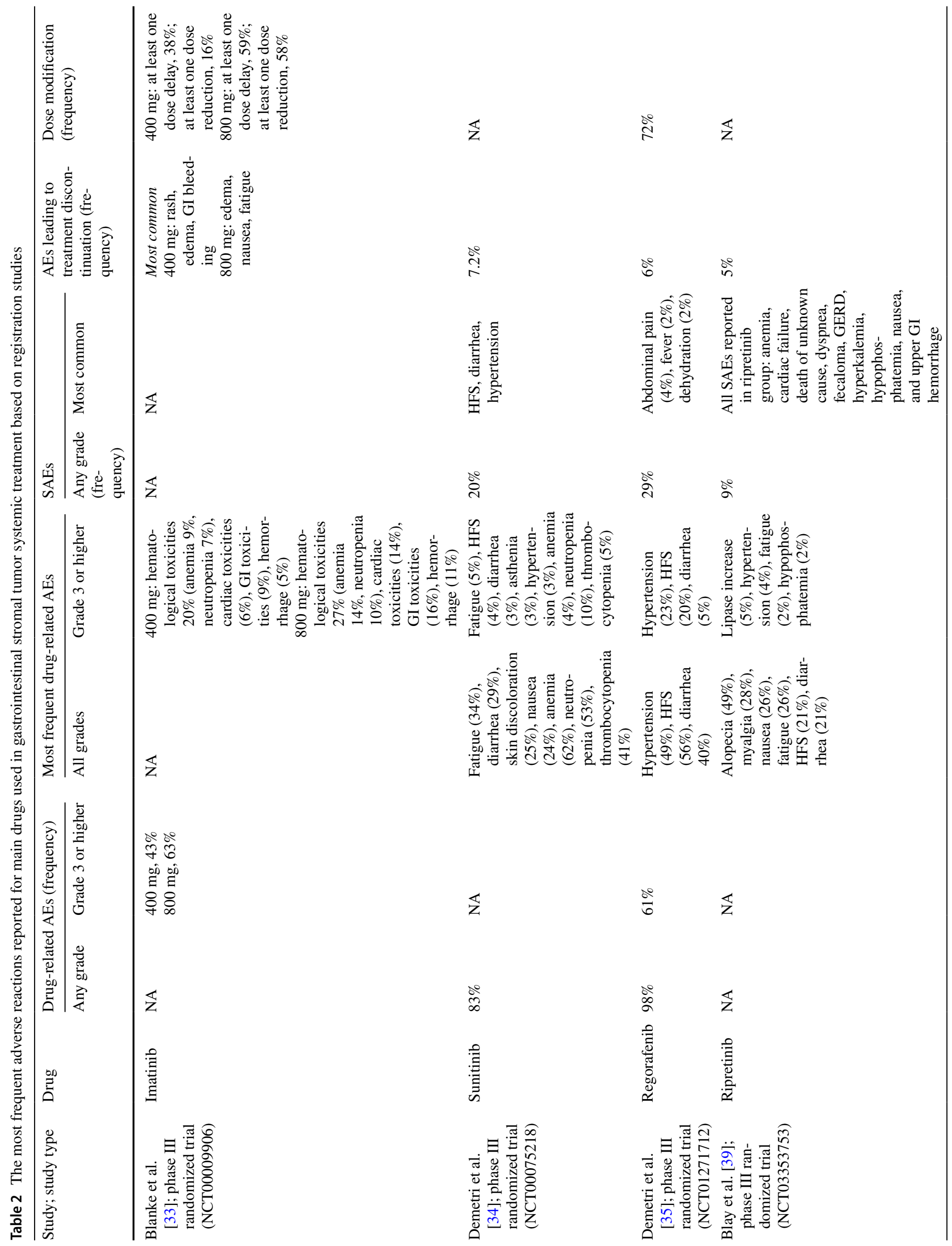




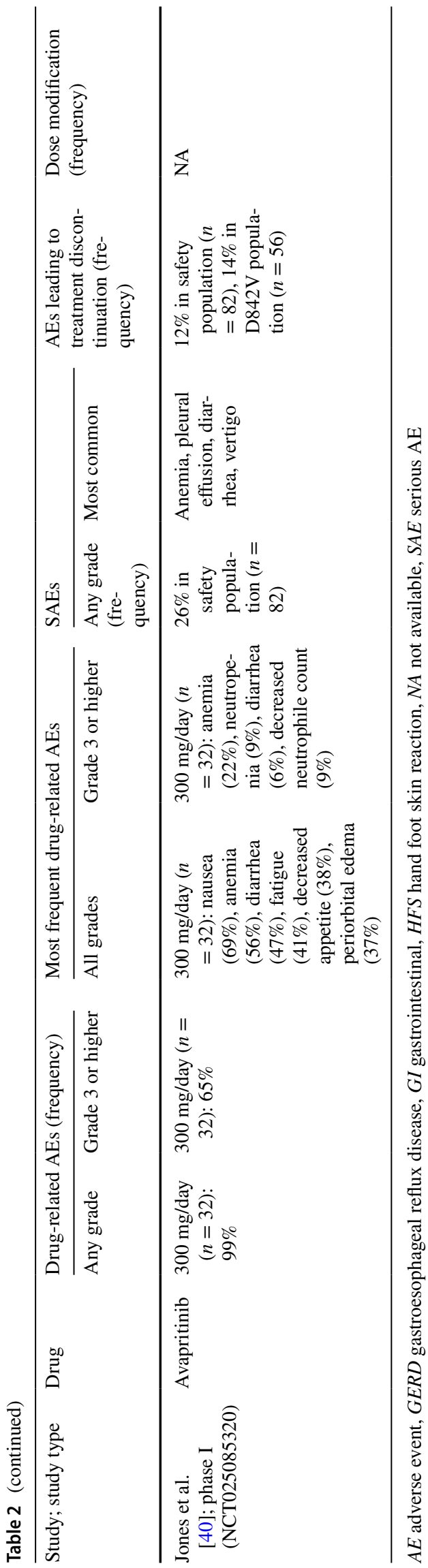

Side effects may improve with prolonged therapy and usually may be managed with appropriate supportive measures. Some patients require drug interruption or reduction. Some studies have reported that the interruption of imatinib is associated with rapid disease progression, and NCCN guidelines for sarcoma recommend the continued use of imatinib treatment at a reduced dose if the side effect recurs after interruption. The summary of product characteristics (SmPC) for imatinib recommend therapy interruption if SAEs occur and return to treatment after improvement [59, 63, 64]. Given the risk of hematological AEs, hematology tests should be undertaken before and regularly during treatment with imatinib. The drug should be stopped if grade 3 neutropenia or thrombocytopenia occurs, until improvement to at least grade 1 (neutrophiles $>1.5 \times 109 / \mathrm{L}$; platelets $>$ $75 \times 109 / \mathrm{L}$ ). It can be resumed at the dose used before the AE. If the event reoccurs, the drug should be interrupted and resumed at the lower dose [84].

Imatinib is mostly metabolized through the liver by cytochrome P450 (CYP)-3A4 and -3A5. Patients with mild, moderate, or severe liver dysfunction should be treated with the minimum recommended dose of $400 \mathrm{mg}$ daily. The dose can be reduced. Liver function should be regularly monitored in all patients. Patients should be careful about possible risk factors for liver damage, such as alcohol use, concomitant medications, and some foods. If bilirubin rises to $>$ $3 \times$ the upper limit of normal (ULN) or liver transaminases rise to $>5 \times$ ULN, imatinib should be interrupted until bilirubin levels return to $<1.5 \times \mathrm{ULN}$ and transaminase levels return to $<2.5 \times$ ULN. Treatment may be continued at a reduced daily dose. The dose should be reduced from 400 to $300 \mathrm{mg} /$ day or from 600 to $400 \mathrm{mg} /$ day, or from 800 to 600 $\mathrm{mg} /$ day [83]. Rare cases of acute liver injury (acute hepatitis) were reported in patients treated with imatinib. In such cases, treatment with prednisolone seems to be useful [89].

During treatment with imatinib, other medicinal products and some foods should be consumed with caution. This refers primarily to protease inhibitors, azole antifungals, certain macrolides, CYP3A4 substrates with a narrow therapeutic window (e.g., cyclosporine, tacrolimus, sirolimus, ergotamine, fentanyl, quinidine), or warfarin and other coumarin derivatives. Grapefruit and grapefruit juice are potent CYP3A4 inhibitors so should be avoided. Products that induce CYP3A4 (e.g., dexamethasone, hypericum perforatum, carbamazepine, phenytoin, rifampicin, phenobarbital) may significantly reduce exposure to imatinib, potentially increasing the risk of therapeutic failure and so should be avoided [84]. Caffeine should also be avoided as imatinib may increase its potency [90].

Proton pump inhibitors (PPIs) are frequently used to manage nausea and dyspepsia in patients receiving imatinib. PPIs can increase gastric $\mathrm{pH}$ and delay gastric emptying and can also antagonize adenosine triphosphate-binding 
transporters, for which imatinib is a known substrate. All these effects can influence the pharmacokinetics of imatinib, potentially decreasing both its absorption and its concentrations. Based on the study by Egorin et al. [91] in 12 healthy subjects, omeprazole may be co-administered with imatinib to treat the gastric AEs without affecting its pharmacokinetics or increasing the risk of tumor progression/relapse [91]. PPIs may decrease the bioavailability and exposure of other TKIs such as sunitinib.

In vitro, imatinib inhibits acetaminophen (paracetamol) $\mathrm{O}$-glucuronidation. This inhibition was not observed in vivo after the administration of imatinib $400 \mathrm{mg}$ and paracetamol $1000 \mathrm{mg}$. Imatinib may increase the plasma levels of acetaminophen when co-administered. The higher doses of imatinib and paracetamol should be used with caution [84].

In patients with impaired renal function, imatinib plasma exposure seems to be higher than that in patients with normal renal function. Prolonged treatment with imatinib may be associated with a clinically significant decline in renal function. Renal function should be assessed before imatinib initiation and closely monitored during therapy, especially in patients with renal function impairment risk factors. If renal function decreases, the appropriate management and treatment should be used according to standard treatment guidelines. Patients with renal dysfunction or who are undergoing dialysis should be treated with the minimum recommended dose of $400 \mathrm{mg}$ daily as a starting dose and carefully monitored. The dose can be reduced if not tolerated or increased if tolerated but lacking efficacy [84].

Skin toxicities are common side effects of treatment with imatinib. It has been shown that imatinib is responsible for grade 1-2 skin rashes in 30-40\% of patients and grade 3-4 skin rashes in $2-5 \%$ of patients. Skin toxicities related to imatinib usually occur shortly after starting treatment but also may develop many months later. The rash is more likely to occur with imatinib doses $>600 \mathrm{mg} /$ day. The typical rash is maculopapular and pruritic and is localized mostly on the forearms, trunk, legs, and face. It seems to be primarily a pharmacological effect rather than a hypersensitivity reaction. Skin rashes are usually self-limiting and can be treated successfully with emollients, topical steroids, and antihistamines, and patients can continue treatment with the same dose of imatinib. Severe cases may require oral steroids and dose interruption until the rash improves to grade 1; such cases may require lower subsequent treatments doses with further attempts to escalate doses. Oral prednisone is usually administered at doses of $0.5-1.0 \mathrm{mg} /$ $\mathrm{kg}$ (or equivalent). Imatinib interruption may be required in rare erythroderma cases, and treatment with oral and topical steroids may be initiated. Vasculitis, Stevens-Johnson syndrome, toxic epidermal necrosis, and hair repigmentation have been observed in rare cases [92]. Imatinib treatment is associated with the risk of phototoxicity, so patients should avoid exposure to direct sunlight and use protective measures such as appropriate clothing and sunscreen with a high sun protection factor [84].

Clinical hypothyroidism cases have been reported in patients who have undergone thyroidectomy and are being treated with levothyroxine replacement during treatment with imatinib. Thyroid-stimulating hormone levels should be closely monitored in such patients.

Fluid retention has been reported during treatment with imatinib. It can take the form of pleural effusion, ascites, pulmonary edema, and rapid weight gain with or without superficial edema. Such AEs can be managed with temporary treatment interruptions, diuretics, and other appropriate supportive care measures. The patients should be weighed regularly. Special care should be taken in patients with cardiac dysfunction [84].

Kim et al. [93] published data from a retrospective study of 403 patients with GIST treated with imatinib. In 15 patients, imaging findings of fluid retention were reported. The most common radiologic sign of fluid retention was subcutaneous edema ( 15 cases), followed by ascites (12 cases), pleural effusion (11 cases), and pericardial effusion (six cases). Two different types of fluid retention were observed: acute/progressive and intermittent/stable. Acute fluid retention occurred mostly early during imatinib treatment initiation or dose escalation; in patients treated with higher doses of imatinib, it was usually severe, often required aggressive management and dose interruption/modification, and improved rapidly after appropriate treatment implementation. The intermittent type of fluid retention occurred any time during treatment with imatinib and required conservative management with or without diuretics and continuing current dose [93].

Cardiac AEs have been reported uncommonly with imatinib treatment. Higher doses are associated with a greater risk of cardiotoxicity. Imatinib-related cardiotoxicity can occur at any age, but the incidence increases with age. Cardiotoxicity may range from asymptomatic mild left ventricular dysfunction to congestive heart failure. In the retrospective analysis of 219 patients treated with imatinib, cardiac AEs were reported in $8.2 \%$ of patients. These events were treated with medical therapy and rarely required imatinib dose reduction or discontinuation [94]. Atallah et al. [95] summarized all cases in the literature of severe cardiac AEs in patients who participated in clinical trials of imatinib and found that systolic heart failure developed in $1.7 \% ; 88 \%$ of patients had preexisting predisposing conditions such as hypertension, diabetes, coronary artery disease, cardiac failure, arrhythmias, and cardiomyopathy [95]. Patients with preexisting cardiac disease or cardiovascular risk factors should be monitored carefully. Any patient with signs or symptoms consistent with cardiac failure should be evaluated, monitored closely, and treated with standard 
medical therapy, including diuretics. In such cases, imatinib should be discontinued/interrupted or the dose reduced [96-98].

Diarrhea is a common side effect of imatinib. The incidence of any-grade diarrhea ranges from 20 to $26 \%$, and the incidence of grade 3-4 diarrhea is $1 \%$. No greater incidence of diarrhea has been reported in older patients receiving imatinib. The current standards of diarrhea management should be applied. It is important to note that diarrhea may more frequently lead to dehydration, electrolyte disturbances, deterioration of kidney function, malnutrition, or even pressure ulcer formation in older people. Grade 1-2 diarrhea with no other complications can be managed conservatively with oral hydration and loperamide. Dietary modifications should be advised and should include the elimination of lactose-containing products and osmolar dietary supplements. The patient should be advised to record the number of stools passed and report fever, dizziness, and other symptoms of life-threatening consequences. Loperamide should be started at an initial dose of $4 \mathrm{mg}$, followed by $2 \mathrm{mg}$ every $4 \mathrm{~h}$ or after every unformed stool. The daily dose of loperamide should not exceed $16 \mathrm{mg}$. Hospitalization, further evaluation, monitoring, and more aggressive treatment are usually required for complications of mild to moderate diarrhea and for severe diarrhea. Such complications may include cramping, nausea, vomiting, dehydration, general condition worsening, fever, sepsis, neutropenia, or bleeding; in severe diarrhea, intravenous hydration, octreotide, antibiotics, and further diagnostic tests, including stool tests for blood and infections, may be required [99].

The study in patients with unresectable or metastatic GIST reported both gastrointestinal and intratumoral hemorrhages. No predisposing factors have been identified as risk factors for either type of bleeding. The standard practices and procedures for monitoring and management should be applied [84].

Nausea and vomiting are prevalent adverse reactions during treatment with imatinib. As per European Society for Medical Oncology (ESMO) 2016 guidelines, imatinib is associated with a moderate risk of nausea and vomiting and, according to NCCN guidelines, has a low emetic risk. The current standards should be applied, taking into consideration drug-drug interactions $[100,101]$.

\subsection{Sunitinib}

As per ESMO and NCCN guidelines, if life-threatening side effects of imatinib are not manageable with maximum supportive treatment, sunitinib should be considered [3, 52]. According to the SmPC for sunitinib, approximately onethird of patients in clinical studies who received sunitinib were aged $\geq 65$ years. No significant differences in safety or efficacy were observed between younger and older patients [102].

To our knowledge, no clinical trials have assessed specifically the safety of sunitinib in elderly patients with GIST. In 2019, Den Hollander et al. [103] published the results of a retrospective analysis of data from 91 patients treated with sunitinib in advanced GIST; 50 patients were aged $\geq 60$ years. In this study, the safety of treatment with sunitinib was consistent with data from the clinical trials. Age $>60$ years was a predictive factor for grade 3 and 4 nonhematological toxicities [103]. In 2013, Duffaud et al. [104] published the data from 71 elderly GIST patients ( $\geq 65$ years) treated with sunitinib in routine clinical practice. This analysis showed similar efficacy as in younger patients. Dose reductions or interruptions were more frequent. Comorbidities were correlated with a higher risk of G3-4 AEs.

Tolerability of sunitinib was assessed in older patients with renal cell cancer. The study included 68 patients with a median age of 74 years. In total, $80.9 \%$ of patients had at least one cardiovascular risk factor, with the most frequently reported being hypertension, alone or associated with other risk factors [105]. Hematological and nonhematological AEs were mostly grade 1-2, and grade 4 AEs included one case of neutropenia, with no febrile complication, and acute myocardial infarction. The most common nonhematological AEs were fatigue (55 cases), mucositis (42 cases), and hypertension (40 cases). Nine cardiac AEs (13.3\%) were reported: grade 4 myocardial infarction and grade 3 congestive heart failure; the others were grade 1-2 and mainly asymptomatic left ventricular fraction decrease and arrhythmias. Generally, doses were reduced in 47 patients (69.2\%), upfront because of frailty $(n=22)$ or after the first $(n=12)$ or subsequent ( $n$ $=13$ ) cycles because of toxicity. No correlation was found between frailty and severe toxicity (grade 3-4) or between frailty and response.

In 2011, Hutson et al. [106] published the pooled data from 1059 patients receiving single-agent sunitinib on the approved $50 \mathrm{mg} /$ day 4-week-on/2-week-off schedule $(n=$ $689)$ or at $37.5 \mathrm{mg}$ continuous once-daily dosing $(n=370)$. In total, $857(81 \%)$ were aged $<70$ years, and $202(19 \%)$ were aged $\geq 70$ years. The efficacy results (PFS and OS) were similar in both groups, as was treatment tolerability. Most treatment-emergent AEs (TEAEs) occurred at similar rates in both age groups. Some AEs were significantly less common in younger patients, including fatigue (59 vs. $69 \%$ ), decreased appetite/decreased weight (29 vs. 53\%), cough ( 20 vs. $29 \%$ ), peripheral edema ( 17 vs. $27 \%$ ), anemia (17 vs. $25 \%$ ), and thrombocytopenia (16 vs. $25 \%$ ). Hand-foot syndrome (HFS) was more common in younger patients (32 vs. $24 \%$ ) [106].

Co-administration of sunitinib with potent CYP3A4 inducers and inhibitors should be avoided [101]. No dose adjustment at the beginning of therapy is required for 
patients with mild renal or liver impairment. Subsequent dose adjustments should be based on individual tolerability. No studies have been conducted in patients with severe liver impairment, so sunitinib is not recommended in patients with severe hepatic insufficiency [102]. AEs related to sunitinib often lead to dose reductions, interruptions, and discontinuations [107]. Hematological AEs, such as anemia, thrombocytopenia, and neutropenia, have been very often observed during therapy with sunitinib. Complete blood counts should be undertaken at the beginning of each treatment cycle for patients receiving sunitinib.

The most common gastrointestinal AEs reported in patients treated with sunitinib were diarrhea, nausea/vomiting, abdominal pain, dyspepsia, and stomatitis, or oral pain. In cases where treatment is required, appropriate management with antiemetic, antidiarrheal, or antacid products should be administered [102]. Grade 2 diarrhea may substantially limit patients' quality of life but rarely requires drug interruption/discontinuation or dose adjustment. For grade 3 diarrhea, sunitinib treatment can be stopped until it reaches grade 1 and then restarted at a reduced dose [84, 107]. Medical management should follow current standards, as described for imatinib-related diarrhea.

Fatigue is a ubiquitous AE during sunitinib therapy, and grade 2 fatigue can severely impact quality of life and ability to undertake daily activities. Management includes ruling out potential alternative causes such as anemia, depression, dehydration, hypothyroidism, or hypercalcemia and undertaking lifestyle changes, including a consistent sleep cycle, maintaining activity levels during the day, avoiding excessive caffeine and alcohol, and ensuring adequate fluid and nutritional intake [84, 107].

Hypothyroidism is a known side effect that all patients may experience with sunitinib therapy. This AE usually does not require treatment interruption and should be treated with thyroid hormone-replacement therapy. Patients should undergo regular monitoring of thyroid function during treatment with sunitinib [107]. Gastrointestinal, respiratory, urinary tract, and brain hemorrhages are known AEs with sunitinib treatment. Routine assessment of bleeding events should include complete blood counts and physical examination. Additionally, patients treated with anticoagulants may be periodically monitored with hematology and coagulation tests and physical examinations [102].

Sunitinib should be interrupted temporarily in patients undergoing major surgical procedures because impaired wound healing has been observed during sunitinib therapy. The decision to resume sunitinib should be based on clinical assessment of recovery from surgery [102].

Oral mucositis, which often occurs during the first month of therapy, requires prompt oral care and dietary modifications. Early use of mouthwashes containing steroids, antibiotics, antifungals, or anesthetics should be considered.
Patients should avoid both mouthwashes containing alcohol and food that is hot, acidic, or spicy and should use soft toothbrushes and sensitive toothpaste. Dose interruptions are rarely necessary, but doses should be modified if grade 3-4 AEs occur; this is usually associated with rapid symptom relief. For grade 3-4 mucositis, treatment can be reassumed if the $\mathrm{AE}$ improves to grade 1 . For grade 3 toxicity, the drug can be reassumed at the previously used dose if toxicity was grade 3 but should be reduced by one level or permanently discontinued according to clinician decision if it was grade 4 [108].

Hypertension has been reported during sunitinib treatment. This AE is a class effect of drugs that target VEGFR and angiogenesis. If severe hypertension cannot be managed with available medication, sunitinib treatment may need to be interrupted. Treatment may be resumed once hypertension is appropriately controlled. For grade 1-2 hypertension, appropriate medical treatment with a calcium antagonist or an angiotensin-converting enzyme inhibitor should be started (diltiazem should be avoided), and sunitinib therapy can be maintained [84, 107]. Other cardiac events, such as heart failure, cardiomyopathy, myocarditis, decreased left ventricular ejection fraction, myocardial ischemia, and myocardial infarction, have also been reported with sunitinib therapy. Patients should be carefully monitored for heart failure signs and symptoms, especially if they have cardiac risk factors or a history of coronary artery disease. In the presence of clinical signs or symptoms of heart failure, sunitinib discontinuation is recommended. In asymptomatic patients with a left ventricular ejection fraction $<50 \%$ and $>20 \%$ below baseline, sunitinib should be interrupted or the dose reduced. Prolonged QT interval and Torsade de Pointes have been observed in patients receiving sunitinib. Therefore, the drug should be used with caution in patients with a known history of QT interval prolongation, patients receiving antiarrhythmics or other drugs that can prolong the QT interval, and patients with relevant preexisting cardiac disease, electrolyte disturbances, or bradycardia [102].

HFS, also known as palmar-plantar erythrodysesthesia, is a cutaneous manifestation associated with sorafenib and sunitinib. Grade 1-2 HFS was reported in 13\% of patients treated with sunitinib and $18 \%$ of patients treated with sorafenib, and grade 3-4 HFS was reported in $4 \%$ of patients treated with sunitinib and $8 \%$ of patients treated with sorafenib [109, 110].

Grade 1-2 rash was reported in $14 \%$ of patients treated with sunitinib and $18 \%$ of patients treated with sorafenib. Grade 3-4 skin rash was reported in $1 \%$ and $2 \%$ of patients treated with sunitinib and sorafenib, respectively. Skin rash may require dose interruption or reduction and supportive treatment, including topical steroids. Alopecia is also a significant AE reported by patients treated with oral TKIs, and 
$30 \%$ of patients treated with sunitinib reported skin discoloration $[109,110]$.

Management of HFS mostly focuses on prevention. The full-body skin examination should include palms and soles. Patients should avoid exposure of their hands and feet to hot water (which may exacerbate symptoms), traumatic activities (especially within the first weeks of treatment), constrictive footwear, and excessive friction on the skin [107]. Patients should also be advised to maintain good hygiene, avoid digging under nails, use gloves for dirty chores, and to apply topical creams and moisturizers to their hands and feet daily from the beginning of therapy. In case of skin overgrowth use of keratolytic may be helpful.

\subsection{Regorafenib}

Treatment with regorafenib frequently requires dose reductions, treatment interruption, or even discontinuation [111]. In the phase II trial of regorafenib in GIST, $82 \%$ of patients required dose modifications [46]. In the phase III GRID study, $72 \%$ of patients required dose modifications, and almost all patients experienced AEs of any grade (98\%). Two grade 5 AEs were considered to be drug related (cardiac arrest and hepatic failure), and $40 \%$ of treatment discontinuations were due to low-grade toxicities [35]. In 2020, Chamberlain et al. [111] published a retrospective analysis of data from 50 patients with GIST treated with regorafenib in Royal Marsden Hospital between March 2013 and September 2018. The main reason for treatment discontinuation was disease progression rather than toxicity. In general, treatment tolerability was similar to that reported in the GRID study. The most frequent grade 3 or higher AEs included HFS ( $n$ $=9)$ and fatigue $(n=7)$. Grade $3-4$ AEs were reported in $46 \%$ of patients $(n=23)$. Dose reductions were required in 19 patients, and eight patients started regorafenib at a lower dose because of comorbidities or concern about an increased individual risk of toxicity [111]. HFS usually begins within the first month of regorafenib treatment, so careful monitoring is vital for early detection and management. Patients should use emollients regularly and avoid skin trauma and pressure. Patients who experience grade 3 or higher HFS can use topical steroids and both topical and oral analgesic agents. In patients experiencing fatigue, any potential deficiencies, such as anemia or vitamin D deficiency, should be corrected, and patients should be advised about graded exercise, sleep hygiene, and appropriate nutritional support. Grade 3 or higher fatigue may require dose modifications [112].

No specific data exist on older/frail patients treated with regorafenib in GIST.

\subsection{Avapritinib}

In a phase III clinical trial with the novel TKI avapritinib in patients with GIST harboring PDGFRA exon 18 mutations, the median age of patients was 64 years (range 29-90). Of the participants, $56 \%$ were exposed for $\geq 6$ months and $44 \%$ were exposed for $>1$ year. The most common adverse reactions occurring in $\geq 10 \%$ of patients treated with avapritinib 300 or $400 \mathrm{mg}$ were edema (all grades in $72 \%$ of patients, grade 3 or higher in $2 \%$ ), nausea (all grades $64 \%$, grade 3 or higher $2.5 \%$ ), fatigue/asthenia (all grades $61 \%$, grade 3 or higher 9\%), cognitive impairment (all grades $48 \%$, grade 3 or higher $4.9 \%$ ), vomiting (all grades $38 \%$, grade 3 or higher $2 \%$ ), and decreased appetite (all grades $38 \%$, grade 3 or higher $2.9 \%$ ). SAEs occurring in at least $1 \%$ of patients who received the drug were anemia (9\%), pleural effusion (3\%), sepsis (3\%), abdominal pain (3\%), acute kidney injury (2\%), vomiting (2\%), gastrointestinal hemorrhage (2\%), pneumonia (1\%), and tumor hemorrhage (1\%). Fatal AEs occurred in $3.4 \%$ of patients. Dose modifications (reductions or interruptions) because of AEs were necessary in $49 \%$ of patients who received the drug, and the median time to dose reduction was 9 weeks. A unique AE, cognitive impairment, was observed in $48 \%$ of patients treated with avapritinib in this study. In the pooled analysis of two clinical trials, cognitive effects, which included memory impairment, confusional state, cognitive disorder, and encephalopathy, were observed in 35 and $48 \%$ of patients receiving avapritinib 300 and 400 $\mathrm{mg} /$ day, respectively. In the 65 patients with cognitive effects in the avapritinib 300-mg/day group, $72 \%$ had grade $1 \mathrm{AEs}$ not affecting daily living, $22 \%$ had grade AEs, and $6 \%$ had grade 3 AEs. No grade 4 cognitive effects were reported. Dose modification effectively managed the grade 2 cognitive impairment. The median time to improvement was 12.0 days for any intervention and 32.5 days for no intervention [113]. The cognitive disturbances may be AEs of special importance in older patients during avapritinib therapy. Data about avapritinib-related toxicities and their treatment among older patients are lacking.

\subsection{Ripretinib}

The second novel drug, ripretinib, was assessed in a phase III study. The median age of patients receiving ripretinib was 59 (range 29-82), and 28 (33\%) patients were aged $\geq 65$ years. Treatment-related TEAEs leading to dose modification were reported in five patients treated with ripretinib, and those leading to treatment discontinuation were reported in four patients (HFS, cardiac failure, death of unknown cause, general physical health deterioration). The most common treatment-related TEAEs, occurring in $\geq 20 \%$ of patients in the ripretinib group, were alopecia, nausea, myalgia, 
fatigue, diarrhea, and HFS. The most common ( $>2 \%)$ grade 3-4 treatment-related TEAEs in the ripretinib group were increased lipase $(n=4)$, hypertension $(n=3)$, hypophosphatemia $(n=2)$, and fatigue $(n=2)$. HFS was grade $1-2$ and managed with routine care. One patient discontinued study treatment due to treatment-related HFS [39]. No data about the incidence of AEs and their management during ripretinib treatment in older patients have been published.

\subsection{Other TKIs}

Data about the incidence and management of AEs in patients with GIST treated with other TKIs are limited.

\subsubsection{Sorafenib}

In the phase II study published by Kindler et al. [54], grade 3-4 AEs included HFS, hypertension, diarrhea, hypophosphatemia, gastrointestinal bleeding, gastrointestinal perforation, thrombosis, and intracranial hemorrhage, and $61 \%$ of patients required dose reductions [54].

In the phase II study conducted by the Korean GIST study group, the most frequently reported AEs were grade 1-2, and most were reversible. The most common AEs of any grade included HFS, skin rash, abdominal pain, and diarrhea. Ten patients required dose reductions or interruption because of intolerance. The most common AEs leading to dose reduction were HFS, rash, hypertension, and diarrhea. No toxicity-related deaths were observed [36].

Neither study separately reported data about treatment tolerability and $\mathrm{AE}$ management in older patients.

\subsubsection{Pazopanib}

In the PAZOGIST study, 76 patients were treated with pazopanib: $72 \%$ experienced pazopanib-related grade 3 or higher AEs, and $26 \%$ of patients reported treatment-related SAEs. The most commonly reported AE was hypertension. Treatment-related AEs occurred in 35\% of participants in the pazopanib group and in $17 \%$ of patients in the best supportive care group. They included five pulmonary embolisms in the pazopanib group and three in the control group [38]. No data about the incidence and management of pazopanibrelated toxicities were published from the PAZOGIST study.

Mourey et al. [114] conducted an open-label phase I doseescalation study in 18 frail patients aged $\geq 75$ years with different metastatic malignancies to find the maximum tolerated dose of pazopanib. They incorporated geriatric assessments and included geriatric criterium for dose-limiting toxicities (DLTs). The starting dose of pazopanib was $400 \mathrm{mg}$, which was escalated to 600 and $800 \mathrm{mg} /$ day. Patients were treated until disease progression. No DLTs were reported at $400 \mathrm{mg} /$ day, one DLT (grade 3 asthenia) was observed at
$600 \mathrm{mg} /$ day, and three DLTs were reported at $800 \mathrm{mg} /$ day. The most frequent treatment-related toxicities were diarrhea, fatigue, and hypertension. Two patients required treatment interruption for more than 2 weeks due to toxicities [114].

\subsubsection{Dasatinib}

Zhou et al. [37] conducted a prospective phase II study and reported that the most frequent AEs were anemia, proteinuria, fatigue, neutropenia, and diarrhea. The main grade 3 AEs included anemia and diarrhea, and $17.2 \%$ of patients experienced grade 1 gastrointestinal bleeding during treatment [37]. Treatment with dasatinib may be complicated by fluid retention, most often manifesting as pleural effusions [51]. No data about AEs in older patients were reported.

\subsubsection{Cabozantinib}

The tolerability of cabozantinib in the CaboGIST study reported by Schöffski et al. [55] was consistent with that observed in previous clinical trials in other indications. AEs were similar to those reported for other TKIs and were generally managed with dose modification and symptomatic treatment. The most common treatment-related AEs, in more than $25 \%$ of patients, were diarrhea, fatigue, hypertension, stomatitis, weight loss, and HFS. Grade 3 or higher diarrhea was reported in $26 \%$ of patients, and HFS was reported in $8 \%$ of patients [55]. No unexpected AEs were observed.

\subsubsection{Ponatinib}

The safety of ponatinib in patients with GIST was assessed in the phase II study by Heinrich et al. [57]. No data about treatment tolerability in older or fragile patients in this study were published. The median age was 59 years. The TEAEs reported in at least $40 \%$ of patients were rash, constipation, fatigue, myalgia, and headache. Serious TEAEs reported in at least two patients were abdominal pain, small bowel obstruction, pneumonia, fatigue, nausea, and vomiting [57]. The results from the POETIG study (NCT03171389) revealed that a lower dose of ponatinib in patients with GIST pretreated with other TKIs was tolerable and had a toxicity profile similar to those of other TKIs used in GIST. Grade 3 and 4 AEs were reported in almost $67 \%$ of patients, and the most frequently observed were pain, hypertension, lipase or gamma-glutamyl transpeptidase elevation, and fever. Six patients experienced SAEs possibly related to ponatinib [58].

\subsubsection{Nilotinib}

In the study by Reichardt et al. [59], AEs were reported in $97.6 \%$ of patients and SAEs in $32.3 \%$ of patients. AEs and 
SAEs were mostly gastrointestinal. The most common AEs in the group treated with nilotinib were abdominal pain, nausea, fatigue, asthenia, anorexia, and anemia. In the nilotinib group, the most frequently reported grade 3-4 AEs were asthenia, increased lipase, abdominal pain, increased alanine aminotransferase, anemia, anorexia, headache, myalgia, and vomiting [59].

\subsubsection{Crenolanib}

Safety data for crenolanib are limited. In a phase II study, grade 3-4 AEs included reversible liver function test elevations and anemia. Increased fluid accumulation in the context of disease progression was observed in a patient with preexisting ascites and pleural effusion. It is important to note that crenolanib reached clinically relevant concentrations despite previous gastrectomy [63].

\section{Discussion}

The majority of patients with cancer are older, and this patient group will increase as life expectancy increases. About $20 \%$ of adults with GIST are aged $>70$ years. As this patient group can be underrepresented in clinical trials because of comorbidities, concomitant medications, limited access to clinical trials, and other reasons, data about the efficacy of therapies used in GIST in older and frail patients are limited. As such, older patients present a particular challenge for clinicians in daily practice. The effectiveness and tolerability of systemic therapies in older patients with GIST seem to be similar to those achieved in younger patients, but some studies have shown that treatment of older patients can be suboptimal. This may be partly the result of inappropriate patient selection for systemic therapies, inadequate management of adverse reactions, insufficient information provided to the patient and caregiver, treatment noncompliance, drug interactions, and so on.

The study definitions of older patients range from 65 to 75 years. For clinical trials, it is defined as 65 years. Chronological age is a poor predictor of treatment tolerability, outcomes, and life expectancy in older patients with cancer. Additional factors, such as social situation, nutritional status, mental and emotional status, and functional status, are significant in older people and may influence the patient's treatment. Considering such elements, chronological age seems to be an inadequate parameter for predicting treatment tolerability. The separate term that is usually related to age is frailty, for which no standard definition exists. It is often recognized as the medical syndrome identifying individuals with decreased physiologic reserve [115] and is more commonly found in older people. A literature review published by Acosta-Benito et al. [116] showed that frailty was associated with an increased risk for mortality and morbidity related to cancer and its treatment and with worse response to treatment. Frailty is often associated with sarcopenia, increased cardiovascular risk, and worse response to infections and treatment [116]. This syndrome should be taken into account when making decisions about treating older patients with cancer. Treating physicians must assess and understand the possible implications of aging and frailty in the treatment process.

Additionally, patient expectations and concerns should always be considered, and the balance between survival benefit and treatment tolerability should also be taken into account. On one hand, the patient faces a potentially lethal disease. On the other, systemic treatment carries a certain risk of serious adverse reactions, especially for older patients who are more likely to have multiple comorbidities and age-related deficits contributing to frailty. Such factors should encourage the clinical oncologist to perform a geriatric assessment. Geriatric assessment improves the decision-making process, reduces toxicity from treatment, and improves older patients' quality of life. In 2018, Shrestha et al. [117] published a systemic literature review of 30 publications to explore patient preferences regarding quality and quantity of life, with interesting results. They found that older age was associated with a preference for quality of life over quantity of life [117]. Appropriate communication and cooperation between physician, patient, and caregiver is crucial when discussing the clinical situation, possible treatment options, treatment adherence, the ability to cope with the disease, and quality of life. Other forms of help, including psychological support, social support, nutritional support, and others, are also necessary. Available studies indicate that patients want to be fully informed and to participate in decision making [118].

In 2019, Martinez-Tapia et al. [118] found that only 23\% of oncologists used standardized geriatric assessment tools to help them make decisions about patients, despite $89 \%$ of them agreeing that cancer care of older adults needed to improve. In 2018, the American Society of Clinical Oncology (ASCO) released guidelines [119] recommending geriatric assessments in patients aged $\geq 65$ years receiving chemotherapy to help oncologists better identify and address potential vulnerabilities in older patients. Geriatric assessment can be divided into various areas and can include physical status, functional status, nutritional status, comorbidities, falls, depression, social support, and cognition [119]. The gold standard of clinical assessment of frailty is the CGA. As this is a time-consuming tool and requires some training, screening tools such as the clinical frailty scale, the frailty phenotype, or the frailty index can be used as a preliminary step to select patients for CGA [120]. 
The prospective randomized study INTEGERATE, presented by Soo et al. [121] at ASCO 2020, was dedicated to assessing health-related quality of life in patients with cancer aged $>70$ years planned for systemic treatment with chemotherapy, targeted therapy, or immunotherapy who underwent CGA and management in comparison with a control group. This study revealed that CGA led to improvements in health-related quality of life, unplanned hospital admissions, and oncological treatment discontinuation [121]. Considering the data discussed, the use of specific tools dedicated to geriatric assessment can also be recommended for older adults with GIST treated with TKIs. In particular, it should be carried out if concerns exist about the patient's ability to tolerate treatment before therapy initiation. If any modifiable abnormalities are identified, they should be appropriately treated.

Data about efficacy and treatment tolerability in older or frail patients with GIST treated with imatinib and other TKIs are limited, and data about the management of toxicities in such patients are also lacking, so the standard rules should be applied, including dose modification, interruption, and discontinuation, and symptomatic treatment. The few publications on the treatment of older patients cited in this review should be taken into account, especially when it comes to predictions of potential toxicity. All of the elements discussed contribute to the personalized treatment that older patients with GIST should receive.

This area should be investigated in future studies, and GIST centers should be encouraged to share their experiences. Prospective clinical trials that include the appropriate number of older and fragile patients are needed to assess the efficacy and safety of targeted therapies in this vulnerable patient population. Additionally, frailty and its importance in the management of patients with GIST should be further investigated.

\section{Conclusions}

The treatment of older patients with GIST has become an increasingly important topic over the last 10 years, mainly because of this patient population's high level of vulnerability. The number of older people will continue to grow as life expectancy increases. GISTs are the most common mesenchymal neoplasms of the gastrointestinal tract, with a median age of diagnosis around 65 years. About $21 \%$ of cases occur in patients aged $>70$ years. Data about the efficacy and safety of TKIs in older patients with GIST are limited. The effectiveness and tolerability results in this group are similar to those in younger patients. Some studies have revealed that treatment of older patients with GIST is suboptimal. Many factors, such as disability, frailty, comorbidities, and drugs used in their treatment, may be challenging for clinical oncologists and their patients. Similarly, data about the incidence and specific management of toxicities in older and fragile patients with GIST are also limited. Toxicities more often lead to treatment discontinuation in this group of patients. The oral route of administration for TKIs and the manageable safety profile can allow for optimization of treatment of older patients with GIST. Maximizing drug exposure with drugs used in GIST treatment whenever possible is essential, as this has a proven influence on treatment efficacy. Imatinib has been the most widely used medical treatment for GIST. Since the introduction of imatinib, the prognosis for patients with GIST has improved significantly, including in older patients. Other TKIs approved in this indication allow further benefits to be gained from medical treatment. An individual approach should be used in the decisionmaking process in older patients with GIST. The general rules for toxicity management should be applied, with an emphasis on the individual approach to each patient. For the management of AEs, dose reduction with appropriate supportive measures is preferred over treatment discontinuation. As discussed, this treatment should be personalized with the intention to use the standard doses whenever possible. The general rules should be applied for older patient selection for targeted therapies with appropriate assessment of patient eligibility, comorbidities, potential interactions with concomitant drugs, patient willingness for treatment, and patient preferences. An essential element that must be included in the treatment strategy is molecular testing and knowledge of the potentially available and most effective therapies, whenever possible. This is mainly to avoid unnecessary and ineffective treatment associated with potential adverse reactions and to maximize treatment outcomes. All these elements contribute to a personalized approach for the older patient, whose well-being is the primary goal of oncological treatment. Before treatment initiation, patients should be assessed for potential risk factors for adverse reactions in particular, including geriatric assessment. Detailed information should be provided to the patient and their caregiver about the context of treatment, clinical situation, potential risks and their management, appropriate dosing, and potential interactions. The clinician should include the patient and caregiver in the decision-making process and consider the patient's preferences, especially in terms of quality of life.

\section{Declarations}

Funding No external funding was used in the preparation of this manuscript.

Conflict of interest Monika Dudzisz-Śledź has received a speaker honorarium from Pierre Fabre, Merck KGaA, Sanofi Aventis, Novartis, and BMS; has received financial support for attending symposia from Novartis; and has consulted for Merck KGaA and Novartis. Piotr Rutkowski has received an educational grant from Pfizer and a speaker 
honorarium from Novartis, Pierre Fabre, Eli Lilly, Merck, Sanofi, MSD, and BMS, and has served as a member of the advisory board for MSD, Pierre Fabre, Sanofi, Merck, Novartis, and BMS. Paweł Teterycz and Elżbieta Bylina have no conflicts of interest that might be relevant to the contents of this manuscript.

Ethics approval Not applicable.

Consent to participate Not applicable.

Consent for publication Not applicable.

Availability of data and material Not applicable.

Author contributions MD-S, EB, PT, PR created the idea for the article. MD-Ś, EB, PT conducted the literature search and data analysis and drafted the work. PR critically revised and supervised the work.

Code availability Not applicable.

Open Access This article is licensed under a Creative Commons Attribution-NonCommercial 4.0 International License, which permits any non-commercial use, sharing, adaptation, distribution and reproduction in any medium or format, as long as you give appropriate credit to the original author(s) and the source, provide a link to the Creative Commons licence, and indicate if changes were made. The images or other third party material in this article are included in the article's Creative Commons licence, unless indicated otherwise in a credit line to the material. If material is not included in the article's Creative Commons licence and your intended use is not permitted by statutory regulation or exceeds the permitted use, you will need to obtain permission directly from the copyright holder. To view a copy of this licence, visit http://creativecommons.org/licenses/by-nc/4.0/.

\section{References}

1. Joensuu H. Gastrointestinal stromal tumor (GIST). Ann Oncol. 2006;17(Suppl 10):x280-286.

2. Rutkowski P, Symonides M, Zdzienicki M, Siedlecki JA. Developments in targeted therapy of advanced gastrointestinal stromal tumors. Recent Pat Anticancer Drug Discov. 2008;3(2):88-99.

3. Casali PG, Abecassis N, Bauer S, Biagini R, Bielack S, Bonvalot $\mathrm{S}$, et al. Gastrointestinal stromal tumours: ESMO-EURACAN clinical practice guidelines for diagnosis, treatment and followup. Ann Oncol. 2018;29:iv68-78.

4. Kindblom LG, Remotti HE, Aldenborg F, Meis-Kindblom JM. Gastrointestinal pacemaker cell tumor (GIPACT): gastrointestinal stromal tumors show phenotypic characteristics of the interstitial cells of Cajal. Am J Pathol. 1998;152:1259-69.

5. Joensuu H, Vehtari A, Riihimäki J, Nishida T, Steigen SE, Brabec $\mathrm{P}$, et al. Risk of recurrence of gastrointestinal stromal tumour after surgery: an analysis of pooled population-based cohorts. Lancet Oncol. 2012;13:265-74.

6. Cerreta F, Eichler H-G, Rasi G. Drug policy for an aging population-the European Medicines Agency's geriatric medicines strategy. N Engl J Med. 2012;367:1972-4.

7. EMA. Refection paper on physical frailty: instruments for baseline characterisation of older populations in clinical trials. 2018. https://www.ema.europa.eu/en/documents/scientific-guideline/ reflection-paper-physical-frailty-instruments-baseline-characteri sation-older-populations-clinical_en.pdf.
8. Perez EA, Livingstone AS, Franceschi D, Rocha-Lima C, Lee DJ, Hodgson N, et al. Current incidence and outcomes of gastrointestinal mesenchymal tumors including gastrointestinal stromal tumors. J Am Coll Surg. 2006;202:623-9.

9. Stiller CA, Trama A, Serraino D, Rossi S, Navarro C, Chirlaque MD, et al. Descriptive epidemiology of sarcomas in Europe: report from the RARECARE project. Eur J Cancer. 2013;49:684-95.

10. Li GZ, Raut CP. Targeted therapy and personalized medicine in gastrointestinal stromal tumors: drug resistance, mechanisms, and treatment strategies. Onco Targets Ther. 2019;12:5123-33.

11. Wada R, Arai H, Kure S, Peng W-X, Naito Z. "Wild type" GIST: clinicopathological features and clinical practice. Pathol Int. 2016;66:431-7.

12. Nannini M, Urbini M, Astolfi A, Biasco G, Pantaleo MA. The progressive fragmentation of the KIT/PDGFRA wild-type (WT) gastrointestinal stromal tumors (GIST). J Transl Med. 2017; $15: 113$.

13. Zsebo KM, Williams DA, Geissler EN, Broudy VC, Martin FH, Atkins HL, et al. Stem cell factor is encoded at the Sl locus of the mouse and is the ligand for the c-kit tyrosine kinase receptor. Cell. 1990;63:213-24.

14. Keshet E, Lyman SD, Williams DE, Anderson DM, Jenkins NA, Copeland NG, et al. Embryonic RNA expression patterns of the $\mathrm{c}$-kit receptor and its cognate ligand suggest multiple functional roles in mouse development. EMBO J. 1991;10:2425-35.

15. Orr-Urtreger A, Avivi A, Zimmer Y, Givol D, Yarden Y, Lonai P. Developmental expression of c-kit, a proto-oncogene encoded by the W locus. Development. 1990;109:911-23.

16. Wozniak A, Rutkowski P, Piskorz A, Ciwoniuk M, Osuch C, Bylina E, et al. Prognostic value of KIT/PDGFRA mutations in gastrointestinal stromal tumours (GIST): Polish Clinical GIST Registry experience. Ann Oncol. 2012;23:353-60.

17. Heinrich MC, Corless CL, Demetri GD, Blanke CD, von Mehren M, Joensuu H, et al. Kinase mutations and imatinib response in patients with metastatic gastrointestinal stromal tumor. J Clin Oncol. 2003;21:4342-9.

18. Hirota S, Isozaki K, Moriyama Y, Hashimoto K, Nishida T, Ishiguro S, et al. Gain-of-function mutations of c-kit in human gastrointestinal stromal tumors. Science. 1998;279:577-80.

19. Vincenzi B, Nannini M, Fumagalli E, Bronte G, Frezza AM, De Lisi D, et al. Imatinib dose escalation versus sunitinib as a second line treatment in KIT exon 11 mutated GIST: a retrospective analysis. Oncotarget. 2016;7:69412-9.

20. Joensuu H, Wardelmann E, Sihto H, Eriksson M, Sundby Hall $\mathrm{K}$, Reichardt A, et al. Effect of KIT and PDGFRA mutations on survival in patients with gastrointestinal stromal tumors treated with adjuvant imatinib: an exploratory analysis of a randomized clinical trial. JAMA Oncol. 2017;3:602-9.

21. Rutkowski P, Ziętek M, Cybulska-Stopa B, Streb J, Głuszek $\mathrm{S}$, Jankowski M, et al. The analysis of 3-year adjuvant therapy with imatinib in patients with high-risk molecular profiled gastrointestinal stromal tumors (GIST) treated in routine practice. Eur J Surg Oncol. 2020:S0748-7983(20)30696-X.

22. Eid S, Turk S, Volkamer A, Rippmann F, Fulle S. KinMap: a web-based tool for interactive navigation through human kinome data. BMC Bioinform. 2017;18:16.

23. Bahlawane C, Schmitz M, Letellier E, Arumugam K, Nicot N, Nazarov PV, et al. Data on quantification of signaling pathways activated by KIT and PDGFRA mutants. Data Brief. 2016;9:828-38.

24. Fathi E, Valipour B, Vietor I, Farahzadi R. An overview of the myocardial regeneration potential of cardiac c-Kit+ progenitor cells via PI3K and MAPK signaling pathways. Future Cardiol. 2020;16:199-209. 
25. Cardoso HJ, Figueira MI, Socorro S. The stem cell factor (SCF)/c-KIT signalling in testis and prostate cancer. J Cell Commun Signal. 2017;11:297-307.

26. Farag S, Somaiah N, Choi H, Heeres B, Wang W-L, van Boven $\mathrm{H}$, et al. Clinical characteristics and treatment outcome in a large multicentre observational cohort of PDGFRA exon 18 mutated gastrointestinal stromal tumour patients. Eur J Cancer. 2017;76:76-83.

27. Avapritinib DS. First approval. Drugs. 2020;80:433-9.

28. Heinrich MC, Jones RL, von Mehren M, Schöffski P, Serrano C, Kang Y-K, et al. Avapritinib in advanced PDGFRA D842Vmutant gastrointestinal stromal tumour (NAVIGATOR): a multicentre, open-label, phase 1 trial. Lancet Oncol. 2020;21:935-46.

29. Kays JK, Sohn JD, Kim BJ, Goze K, Koniaris LG. Approach to wild-type gastrointestinal stromal tumors. Transl Gastroenterol Hepatol. 2018;3:92.

30. Antonescu CR, Besmer P, Guo T, Arkun K, Hom G, Koryotowski $\mathrm{B}$, et al. Acquired resistance to imatinib in gastrointestinal stromal tumor occurs through secondary gene mutation. Clin Cancer Res. 2005;11:4182-90.

31. Astolfi A, Pantaleo MA, Indio V, Urbini M, Nannini M. The emerging role of the FGF/FGFR pathway in gastrointestinal stromal tumor. Int J Mol Sci. 2020;21(9):3313.

32. Verweij J, Casali PG, Zalcberg J, LeCesne A, Reichardt P, Blay $\mathrm{J}-\mathrm{Y}$, et al. Progression-free survival in gastrointestinal stromal tumours with high-dose imatinib: randomised trial. Lancet. 2004;364:1127-34.

33. Blanke CD, Rankin C, Demetri GD, Ryan CW, von Mehren $\mathrm{M}$, Benjamin RS, et al. Phase III randomized, intergroup trial assessing imatinib mesylate at two dose levels in patients with unresectable or metastatic gastrointestinal stromal tumors expressing the kit receptor tyrosine kinase: S0033. J Clin Oncol. 2008;26:626-32.

34. Demetri GD, van Oosterom AT, Garrett CR, Blackstein ME, Shah MH, Verweij J, et al. Efficacy and safety of sunitinib in patients with advanced gastrointestinal stromal tumour after failure of imatinib: a randomised controlled trial. Lancet. 2006;368:1329-38.

35. Demetri GD, Reichardt P, Kang Y-K, Blay J-Y, Rutkowski $\mathrm{P}$, Gelderblom $\mathrm{H}$, et al. Efficacy and safety of regorafenib for advanced gastrointestinal stromal tumours after failure of imatinib and sunitinib (GRID): an international, multicentre, randomised, placebo-controlled, phase 3 trial. Lancet. 2013;381:295-302.

36. Park SH, Ryu MH, Ryoo BY, Im SA, Kwon HC, Lee SS, et al. Sorafenib in patients with metastatic gastrointestinal stromal tumors who failed two or more prior tyrosine kinase inhibitors: a phase II study of Korean gastrointestinal stromal tumors study group. Investig New Drugs. 2012;30:2377-83.

37. Zhou Y, Zhang X, Wu X, Zhou Y, Zhang B, Liu X, et al. A prospective multicenter phase II study on the efficacy and safety of dasatinib in the treatment of metastatic gastrointestinal stromal tumors failed by imatinib and sunitinib and analysis of NGS in peripheral blood. Cancer Med. 2020;9(17):6225-33.

38. Mir O, Cropet C, Toulmonde M, Cesne AL, Molimard M, Bompas E, et al. Pazopanib plus best supportive care versus best supportive care alone in advanced gastrointestinal stromal tumours resistant to imatinib and sunitinib (PAZOGIST): a randomised, multicentre, open-label phase 2 trial. Lancet Oncol. 2016;17:632-41.

39. Blay J-Y, Serrano C, Heinrich MC, Zalcberg J, Bauer S, Gelderblom $\mathrm{H}$, et al. Ripretinib in patients with advanced gastrointestinal stromal tumours (INVICTUS): a double-blind, randomised, placebo-controlled, phase 3 trial. Lancet Oncol. 2020;21:923-34.

40. Jones RL, Serrano C, von Mehren M, George S, Heinrich M, Kang Y-K, et al. 1621MO Long-term efficacy, tolerability and overall survival in patients (pts) with unresectable or metastatic (U/M) PDGFRA D842V-mutant gastrointestinal stromal tumour (GIST) treated with avapritinib: NAVIGATOR phase I trial update. Ann Oncol. 2020;31:S973.

41. Bauer S, George S, Kang Y-K, Tap WD, Zhou T, Picazio N, et al. VOYAGER: An open-label, randomised, phase III study of avapritinib vs. regorafenib in patients (pts) with locally advanced (adv) metastatic or unresectable gastrointestinal stromal tumour (GIST). Annals of Oncology. 2018;29:viii595.

42. Rutkowski P, Andrzejuk J, Bylina E, Osuch C, Switaj T, Jerzak vel Dobosz A, et al. What are the current outcomes of advanced gastrointestinal stromal tumors: who are the long-term survivors treated initially with imatinib? Med Oncol. 2013;30:765.

43. Reichardt P, Kang Y-K, Rutkowski P, Schuette J, Rosen LS, Seddon B, et al. Clinical outcomes of patients with advanced gastrointestinal stromal tumors: safety and efficacy in a worldwide treatment-use trial of sunitinib. Cancer. 2015;121:1405-13.

44. George S, Blay JY, Casali PG, Le Cesne A, Stephenson P, Deprimo SE, et al. Clinical evaluation of continuous daily dosing of sunitinib malate in patients with advanced gastrointestinal stromal tumour after imatinib failure. Eur J Cancer. 2009;45:1959-68.

45. Heinrich MC, Maki RG, Corless CL, Antonescu CR, Harlow A, Griffith D, et al. Primary and secondary kinase genotypes correlate with the biological and clinical activity of sunitinib in imatinib-resistant gastrointestinal stromal tumor. J Clin Oncol. 2008;26:5352-9.

46. George S, Wang Q, Heinrich MC, Corless CL, Zhu M, Butrynski JE, et al. Efficacy and safety of regorafenib in patients with metastatic and/or unresectable GI stromal tumor after failure of imatinib and sunitinib: a multicenter phase II trial. J Clin Oncol. 2012;30:2401-7.

47. Nemunaitis J, Bauer S, Blay J-Y, Choucair K, Gelderblom H, George S, et al. Intrigue: Phase III study of ripretinib versus sunitinib in advanced gastrointestinal stromal tumor after imatinib. Future Oncol. 2020;16:4251-64.

48. AYVAKYT (avapritinib) SMPC [Internet]. https://www.ema. europa.eu/en/documents/product-information/ayvakyt-eparproduct-information_en.pdf. Cited 2020 Dec 17.

49. AYVAKYT (avapritinib) prescribing information. [Internet]. Available from: https://www.accessdata.fda.gov/drugsatfda _docs/label/2020/212608s000lbl.pdf. Cited 2020 Dec 17.

50. Farag S, Smith MJ, Fotiadis N, Constantinidou A, Jones RL. Revolutions in treatment options in gastrointestinal stromal tumours (GISTs): the latest updates. Curr Treat Opt Oncol. 2020;21:55.

51. Cortes J, Kim D-W, Raffoux E, Martinelli G, Ritchie E, Roy L, et al. Efficacy and safety of dasatinib in imatinib-resistant or -intolerant patients with chronic myeloid leukemia in blast phase. Leukemia. 2008;22:2176-83.

52. National Comprehensive Cancer Network. GIST (Version 1.2021) [Internet]. https://www.nccn.org/professionals/physi cian_gls/pdf/gist.pdf.

53. Brzozowska M, Wierzba W, Szafraniec-Buryło S, Czech M, Połowinczak-Przybyłek J, Potemski P, et al. Real-world evidence of patient outcome following treatment of advanced gastrointestinal stromal tumor (GIST) with imatinib, sunitinib, and sorafenib in publicly funded health care in Poland. Med Sci Monit Int Med J Exp Clin Res. 2019;25:3846-53.

54. Kindler HL, Campbell NP, Wroblewski K, Maki RG, D'Adamo DR, Chow WA, et al. Sorafenib (SOR) in patients (pts) with imatinib (IM) and sunitinib (SU)-resistant (RES) gastrointestinal stromal tumors (GIST): Final results of a University of Chicago Phase II Consortium trial. J Clin Oncol. 2011;29:10009-10009.

55. Schöffski P, Mir O, Kasper B, Papai Z, Blay J-Y, Italiano A, et al. Activity and safety of the multi-target tyrosine kinase inhibitor cabozantinib in patients with metastatic gastrointestinal stromal 
tumour after treatment with imatinib and sunitinib: European Organisation for Research and Treatment of Cancer phase II trial 1317 'CaboGIST.' Eur J Cancer. 2020;134:62-74.

56. Garner AP, Gozgit JM, Anjum R, Vodala S, Schrock A, Zhou $\mathrm{T}$, et al. Ponatinib inhibits polyclonal drug-resistant KIT oncoproteins and shows therapeutic potential in heavily pretreated gastrointestinal stromal tumor (GIST) patients. Clin Cancer Res. 2014;20:5745-55.

57. Heinrich MC, von Mehren M, Demetri GD, Fletcher JA, Sun JG, Kerstein D, et al. Ponatinib efficacy and safety in patients (pts) with advanced gastrointestinal stromal tumors (GIST) after tyrosine kinase inhibitor (TKI) failure: results from a phase 2 study. J Clin Oncol. 2015;33:10535-10535.

58. Falkenhorst J, Hamacher R, Reichardt P, Ivanyi P, Kasper B, Hohenberger $\mathrm{P}$, et al. Lower-dosing ponatinib in pre-treated GIST: results of the POETIG phase II trial. J Clin Oncol. 2020;38:11536-11536.

59. Reichardt P, Blay J-Y, Gelderblom H, Schlemmer M, Demetri GD, Bui-Nguyen B, et al. Phase III study of nilotinib versus best supportive care with or without a TKI in patients with gastrointestinal stromal tumors resistant to or intolerant of imatinib and sunitinib. Ann Oncol. 2012;23:1680-7.

60. Blay J-Y, Shen L, Kang Y-K, Rutkowski P, Qin S, Nosov D, et al. Nilotinib versus imatinib as first-line therapy for patients with unresectable or metastatic gastrointestinal stromal tumours (ENESTg1): a randomised phase 3 trial. Lancet Oncol. 2015;16:550-60.

61. Montemurro M, Schöffski P, Reichardt P, Gelderblom H, Schütte J, Hartmann JT, et al. Nilotinib in the treatment of advanced gastrointestinal stromal tumours resistant to both imatinib and sunitinib. Eur J Cancer. 2009;45:2293-7.

62. Cauchi C, Somaiah N, Engstrom PF, Litwin S, Lopez M, Lee $\mathrm{J}$, et al. Evaluation of nilotinib in advanced GIST previously treated with imatinib and sunitinib. Cancer Chemother Pharmacol. 2012;69:977-82.

63. Heinrich MC, Griffith D, McKinley A, Patterson J, Presnell A, Ramachandran A, et al. Crenolanib inhibits the drugresistant PDGFRA D842V mutation associated with imatinibresistant gastrointestinal stromal tumors. Clin Cancer Res. 2012;18:4375-84.

64. von Mehren M, Tetzlaff ED, Macaraeg M, Davis J, Agarwal V, Ramachandran A, et al. Dose escalating study of crenolanib besylate in advanced GIST patients with PDGFRA D842V activating mutations. J Clin Oncol. 2016;34:11010-11010.

65. Blay J-Y, Heinrich MC, Hohenberger P, Casali PG, Rutkowski P, Serrano-Garcia C, et al. A randomized, double-blind, placebocontrolled, phase III study of crenolanib in advanced or metastatic GIST patients bearing a D842V mutation in PDGFRA: the CrenoGIST study. J Clin Oncol. 2017;35:TPS11080-TPS11080.

66. Chen Z, Lin R-M, Bai Y-K, Zhang Y. Establishment and verification of prognostic nomograms for patients with gastrointestinal stromal tumors: a SEER-based study. Biomed Res Int. 2019;2019:8293261.

67. Kramer K, Knippschild U, Mayer B, Bögelspacher K, Spatz H, Henne-Bruns D, et al. Impact of age and gender on tumor related prognosis in gastrointestinal stromal tumors (GIST). BMC Cancer. 2015;15:57.

68. Farag S, van Coevorden F, Sneekes E, Grunhagen DJ, Reyners AKL, Boonstra PA, et al. Elderly patients with gastrointestinal stromal tumour (GIST) receive less treatment irrespective of performance score or comorbidity-a retrospective multicentre study in a large cohort of GIST patients. Eur J Cancer. 2017;86:318-25.

69. Yoo C, Ryu M-H, Nam B-H, Ryoo B-Y, Demetri GD, Kang Y-K. Impact of imatinib rechallenge on health-related quality of life in patients with TKI-refractory gastrointestinal stromal tumours:
Sub-analysis of the placebo-controlled, randomised phase III trial (RIGHT). Eur J Cancer. 2016;52:201-8.

70. Poole CD, Connolly MP, Chang J, Currie CJ. Health utility of patients with advanced gastrointestinal stromal tumors (GIST) after failure of imatinib and sunitinib: findings from GRID, a randomized, double-blind, placebo-controlled phase III study of regorafenib versus placebo. Gastric Cancer. 2015;18:627-34.

71. Rutkowski P, Bylina E, Lugowska I, Teterycz P, Klimczak A, Streb J, et al. Treatment outcomes in older patients with advanced gastrointestinal stromal tumor (GIST). J Geriatr Oncol. 2018;9:520-5.

72. Molinas Mandel N, Selcukbiricik F, Kanitez M, Yalcin S, Tural D, Erdamar S, et al. Clinical and pathological characteristics and their effect on survival in elderly patients with gastrointestinal stromal tumors. J BUON. 2016;21:360-5.

73. Italiano A, Saada E, Cioffi A, Poulette S, Bouchet S, Molimard $\mathrm{M}$, et al. Treatment of advanced gastrointestinal stromal tumors in patients over 75 years old: clinical and pharmacological implications. Targ Oncol. 2013;8:295-300.

74. Tham C-K, Poon DY-H, Li H-H, Tan M-H, Choo S-P, Foo K-F. Gastrointestinal stromal tumour in the elderly. Crit Rev Oncol Hematol. 2009;70:256-61.

75. Yang Z, Feng X, Zhang P, Chen T, Qiu H, Zhou Y, et al. Clinicopathological outcomes and prognosis of elderly patients $(\geq$ 65 years) with gastric gastrointestinal stromal tumors (GISTs) undergoing curative-intent resection: a multicenter data review. J Gastrointest Surg. 2019;23:904-13.

76. Smith MJ, Smith HG, Mahar AL, Law C, Ko Y-J. The impact of additional malignancies in patients diagnosed with gastrointestinal stromal tumors. Int J Cancer. 2016;139:1744-51.

77. Vassos N, Agaimy A, Hohenberger W, Croner RS. Coexistence of gastrointestinal stromal tumours (GIST) and malignant neoplasms of different origin: Prognostic implications. Int J Surg. 2014;12:371-7.

78. Marosi C, Köller M. Challenge of cancer in the elderly. ESMO Open. 2016;1:e000020.

79. Repetto L, Fratino L, Audisio RA, Venturino A, Gianni W, Vercelli M, et al. Comprehensive geriatric assessment adds information to Eastern Cooperative Oncology Group performance status in elderly cancer patients: an Italian Group for Geriatric Oncology Study. J Clin Oncol. 2002;20:494-502.

80. Lebreton C, Cantarel C, Toulza E, Desgrippes R, Bozec Lemoal L, Saada E, et al. Predicting severe toxicity of targeted therapies in elderly patients with cancer (PreToxE): A multicenter, prospective, and retrospective study. J Clin Oncol. 2019;37:11550-11550.

81. Abdel-Qadir H, Ethier J-L, Lee DS, Thavendiranathan P, Amir E. Cardiovascular toxicity of angiogenesis inhibitors in treatment of malignancy: a systematic review and meta-analysis. Cancer Treat Rev. 2017;53:120-7.

82. Hamnvik R, Choueiri TK, Turchin A, McKay RR, Goyal L, Davis M, Kaymakcalan MD, Williams JS (2015) Clinical risk factors for the development of hypertension in patients treated with inhibitors of the VEGF signaling pathway. Cancer 121(2):311-9.

83. Teng JFT, Mabasa VH, Ensom MHH. The role of therapeutic drug monitoring of imatinib in patients with chronic myeloid leukemia and metastatic or unresectable gastrointestinal stromal tumors. Ther Drug Monit. 2012;34:85-97.

84. Glivec (imatinib) summary of product characteristics, last update 13 Nov 2020. https://www.ema.europa.eu/en/documents/produ ct-information/glivec-epar-product-information_en.pdf.

85. Schmidli H, Peng B, Riviere G-J, Capdeville R, Hensley M, Gathmann I, et al. Population pharmacokinetics of imatinib mesylate in patients with chronic-phase chronic myeloid leukaemia: results of a phase III study. Br J Clin Pharmacol. 2005;60:35-44. 
86. de Wit D, Guchelaar H-J, den Hartigh J, Gelderblom H, van Erp NP. Individualized dosing of tyrosine kinase inhibitors: are we there yet? Drug Discov Today. 2015;20:18-36.

87. D’Ambrosio L, Ponzetti A, Lista P, Bombaci S, Palesandro E, Galizia D, et al. Imatinib mesylate (IM) therapy in elderly patients affected by advanced gastrointestinal stromal tumor (GIST). J Clin Oncol. 2011;29:e20514-e20514.

88. National Comprehensive Cancer Network. Soft tissue sarcoma (Version 2.2020) [Internet]. https://www.nccn.org/professionals/ physician_gls/PDF/sarcoma.pdf. Cited 6 Oct 2020.

89. Tonyali O, Coskun U, Yildiz R, Karakan T, Demirci U, Akyurek $\mathrm{N}$, et al. Imatinib mesylate-induced acute liver failure in a patient with gastrointestinal stromal tumors. Med Oncol. 2010;27:768-73.

90. O’Brien SG, Meinhardt P, Bond E, Beck J, Peng B, Dutreix C, et al. Effects of imatinib mesylate (STI571, Glivec) on the pharmacokinetics of simvastatin, a cytochrome p450 3A4 substrate, in patients with chronic myeloid leukaemia. Br J Cancer. 2003;89:1855-9.

91. Egorin MJ, Shah DD, Christner SM, Yerk MA, Komazec KA, Appleman LR, et al. Effect of a proton pump inhibitor on the pharmacokinetics of imatinib. Br J Clin Pharmacol. 2009;68:370-4.

92. Scott LC, White JD, Reid R, Cowie F. Management of skin toxicity related to the use of imatinib mesylate (STI571, Glivectrade mark) for advanced stage gastrointestinal stromal tumours. Sarcoma. 2005;9:157-60.

93. Kim KW, Shinagare AB, Krajewski KM, Pyo J, Tirumani SH, Jagannathan JP, et al. Fluid retention associated with imatinib treatment in patients with gastrointestinal stromal tumor: quantitative radiologic assessment and implications for management. Korean $\mathbf{J}$ Radiol. 2015;16:304-13.

94. Trent JC, Patel SS, Zhang J, Araujo DM, Plana J-C, Lenihan DJ, et al. Rare incidence of congestive heart failure in gastrointestinal stromal tumor and other sarcoma patients receiving imatinib mesylate. Cancer. 2010;116:184-92.

95. Atallah E, Durand J-B, Kantarjian H, Cortes J. Congestive heart failure is a rare event in patients receiving imatinib therapy. Blood. 2007;110:1233-7.

96. Distler JHW, Distler O. Cardiotoxicity of imatinib mesylate: an extremely rare phenomenon or a major side effect? Ann Rheum Dis. 2007;66:836.

97. Kerkelä R, Grazette L, Yacobi R, Iliescu C, Patten R, Beahm $\mathrm{C}$, et al. Cardiotoxicity of the cancer therapeutic agent imatinib mesylate. Nat Med. 2006;12:908-16.

98. Ran H-H, Zhang R, Lu X-C, Yang B, Fan H, Zhu H-L. Imatinibinduced decompensated heart failure in an elderly patient with chronic myeloid leukemia: case report and literature review. J Geriatr Cardiol. 2012;9:411-4

99. Bossi P, Antonuzzo A, Cherny NI, Rosengarten O, Pernot S, Trippa $F$, et al. Diarrhoea in adult cancer patients: ESMO clinical practice guidelines. Ann Oncol. 2018;29:126-42.

100. Walsh D, Davis M, Ripamonti C, Bruera E, Davies A, Molassiotis A. 2016 Updated MASCC/ESMO consensus recommendations: Management of nausea and vomiting in advanced cancer. Support Care Cancer. 2017;25:333-40.

101. National Comprehensive Cancer Network. Antiemesis (Version 2.2020) [Internet]. https://www.nccn.org/professionals/physician_ gls/pdf/antiemesis.pdf. Cited 6 Oct 2020.

102. Sutent (sunitinib), summary of product characteristics, last update 27 Nov 2019. https://www.ema.europa.eu/en/documents/productinformation/sutent-epar-product-information_en.pdf.

103. Den Hollander D, Van der Graaf WTA, Desar IME, Le Cesne A. Predictive factors for toxicity and survival of second-line sunitinib in advanced gastrointestinal stromal tumours (GIST). Acta Oncol. 2019;58:1648-54.

104. Duffaud F, Ray-Coquard I, Marchal F, et al. Clinical experience with sunitinib (SU) in patients over age 65 with metastatic gastrointestinal stromal tumors (GIST): a retrospective study from the French Sarcoma Group (FSG). J Clin Oncol. 2013;31(suppl): 10546.

105. Brunello A, Basso U, Sacco C, Sava T, De Vivo R, Camerini A, et al. Safety and activity of sunitinib in elderly patients ( $\geq 70$ years) with metastatic renal cell carcinoma: a multicenter study. Ann Oncol. 2013;24:336-42.

106. Hutson TE, Bukowski RM, Rini BI, Gore ME, Larkin JMG, Figlin RA, et al. A pooled analysis of the efficacy and safety of sunitinib in elderly patients (pts) with metastatic renal cell carcinoma (mRCC). J Clin Oncol. 2011;29:4604-4604.

107. Sehdev S. Sunitinib toxicity management—a practical approach. Can Urol Assoc J. 2016;10:S248-51.

108. Valle JW, Faivre S, Hubner RA, Grande E, Raymond E. Practical management of sunitinib toxicities in the treatment of pancreatic neuroendocrine tumors. Cancer Treat Rev. 2014;40:1230-8.

109. Adams VR, Leggas M. Sunitinib malate for the treatment of metastatic renal cell carcinoma and gastrointestinal stromal tumors. Clin Ther. 2007;29:1338-53.

110. Strumberg D, Clark JW, Awada A, Moore MJ, Richly H, Hendlisz A, et al. Safety, pharmacokinetics, and preliminary antitumor activity of sorafenib: a review of four phase I trials in patients with advanced refractory solid tumors. Oncologist. 2007;12:426-37.

111. Chamberlain F, Farag S, Williams-Sharkey C, Collingwood C, Chen L, Mansukhani S, et al. Toxicity management of regorafenib in patients with gastro-intestinal stromal tumour (GIST) in a tertiary cancer centre. Clin Sarcoma Res. 2020;10:1.

112. Krishnamoorthy SK, Relias V, Sebastian S, Jayaraman V, Saif MW. Management of regorafenib-related toxicities: a review. Ther Adv Gastroenterol. 2015;8:285-97.

113. Maggo S, Dubey AP, Dhull P, Singh NK. Avapritinib: novel hope for patients with metastatic gist with PDGFRA exon 18 mutation. Int J Basic Clin Pharmacol. 2020;9:1175-9.

114. Mourey L, Ravaud A, Digue L, Cabarrou B, Gomez-Roca CA, Valentin T, et al. VOTRAGE study pazopanib in a population of "frail" elderly patients after geriatric assessment: A phase I study with geriatric criteria. Ann Oncol. 2018;29:viii319.

115. Huisingh-Scheetz M, Walston J. How should older adults with cancer be evaluated for frailty? J Geriatr Oncol. 2017;8:8-15.

116. Acosta-Benito MA, Garcia-Pliego RA, Barrio-Cortes J, RodriguezFernandez V. Frailty as a prognosis factor in cancer for the elderly. Int J Cancer Clin Res. 2019;6:118.

117. Shrestha A, Martin C, Burton M, Walters S, Collins K, Wyld L. Quality of life versus length of life considerations in cancer patients: a systematic literature review. Psychooncology. 2019;28:1367-80.

118. Martinez-Tapia C, Canoui-Poitrine F, Caillet P, Bastuji-Garin S, Tournigand C, Assaf E, et al. Preferences for surrogate designation and decision-making process in older versus younger adults with cancer: a comparative cross-sectional study. Patient Educ Couns. 2019;102:429-35.

119. Mohile SG, Dale W, Somerfield MR, Schonberg MA, Boyd CM, Burhenn PS, et al. Practical assessment and management of vulnerabilities in older patients receiving chemotherapy: ASCO guideline for geriatric oncology. J Clin Oncol. 2018;36:2326-47.

120. O'Donovan A, Leech M. Personalised treatment for older adults with cancer: the role of frailty assessment. Tech Innov Patient Support Radiat Oncol. 2020;16:30-8.

121. Soo W-K, King M, Pope A, Parente P, Darzins P, Davis ID. Integrated geriatric assessment and treatment (INTEGERATE) in older people with cancer planned for systemic anticancer therapy. J Clin Oncol. 2020;38:12011-12011. 